\title{
Verfassungsgebung im GUS-Land - Erwachen aus sozialistischem Schlaf oder Aufwecken der sozialistischen Schläfer ${ }^{1}$
}

\author{
Von Alexander Blankenagel
}

\section{A. Zum konkreten Stand und zur politischen Situation der Verfassungsgebung}

In den Nachfolgestaaten der UdSSR "verfasselt" es allerorten: Einige Staaten haben in relativ schmerzlosen Prozessen sich schon neue Verfassungen gegeben; die Russische Föderation als der wichtigste Nachfolgestaat der UdSSR hat soeben das gleiche in einem mehr als schmerzhaften ProzeB vollbracht (dieses Wort setzt sich angesichts der konkreten Geschehensabläufe dem Euphemieverdacht aus). In anderen Ländern arbeitet man seit drei Jahren an neuen Verfassungen, ohne daß die Parlamente große Neigungen zeigen würden, diese Verfassungen auch zu beschließen; schließlich gibt es auch Beispiele wie Georgien, in denen alle Pläne, eine neue Verfassung auszuarbeiten (in diesem Fall sogar an bürgerlichen Vorbildern aus den 20er Jahren) in den Wirren des Bürgerkriegs vorläufig gescheitert sind. 2 Wir wollen uns im folgenden in vergleichender Betrachtung den Verfassungen und Verfassungsprojekten einiger der Nachfolgestaaten der UdSSR mit der spezifischen Neugierde widmen, herauszufinden, welche Inhalte dort überhaupt in den Verfassungen festgeschrieben werden bzw. festgeschrieben werden sollen und ob sich irgendwelche verallgemeinerungsfähigen Besonderheiten postsozialistischer Verfassungsgebung erkennen lassen. Die gewählten Beispiele sollen dabei jedes auf seine Art in besonderer Weise typisch für eine bestimmte Situation und typisch für bestimmte Probleme sein: Es geht um die Russische Föderation, die Ukraine, Weißrußland, Estland, Kasachstan und Usbekistan. Die Wahl der Russischen Föderation spricht für sich selbst; an diesem Land führt - buchstäblich und übertragen - kein Weg vorbei. Die Ukraine und Weißrußland stehen für zwei slawische

1 Ausgearbeitete Fassung eines als dritte "Herbert-Krüger-Gedächtnisvorlesung" auf der Jahrestagung 1993 des Arbeitskreises für überseeische Veffassungsvergleichung in Berlin gehaltenen Vortrags.

2 Das georgische Verfassungsprojekt ist eine von der Soros-Foundation gefördente Unternehmung, bei der zunächst jüngere georgische Juristen an der Law-School der Universität von Chicago vorbereitet wurden und dann auf einer Reihe von Konferenzen als Vorbereitung Problernaspekte der zu schaffenden Verfassung mit westlichen Experten diskutiert wurden. Am 16.2.1993 beschloß das Parlament (Entscheidung Nr. 149), eine Verfassungskommission einzusetzen; das Verfassungsprojekt sollte im Oktober / November auf einer weiteren Konferenz vorgestellt und mit westlichen Rechtswissenschaftlem diskutiert werden. Der sich verschärfende Bürgerkrieg führte zu einer Vertagung der Vorstellung des Verfassungsprojektes. 
Nachfolgestaaten, von denen die Ukraine volens, Weißrußland aber eher nolens aus dem Bundesstaat UdSSR ausschieden; Estland steht für ein nach Westeuropa (und nach Finnland) schauendes europäisches und nicht-slawisches Land; Kasachstan und Usbekistan schließlich repräsentieren die Vielfalt der asiatischen Republiken, Kasachstan als nicht islamischer, relativ reicher Staat mit einem großen Anteil von Russen in der Bevölkerung und Usbekistan wiederum als stark islamisches Land mit wenig russischer Bevölkerung und einer durch die Baumwoll-Monokultur völlig abgewirtschafteten Volkswirtschaft.

Von unseren Beispielländern haben Kasachstan und Usbekistan den Prozeß der Verfassungsgebung im letzten Jahre abgeschlossen: In beiden Ländem war die Verfassungsgebung relativ unkompliziert, jeweils nur mit einem Verfassungsentwurf und relativ intakten (Kasachstan) oder gesellschaftlichen (Usbekistan) Strukturen, die wohl aus unterschiedlichen Gründen für die reibungslose Verabschiedung einer Verfassung sorgten. 3 Problematischer verläuft der Prozeß in Weißrußland und in der Ukraine. In beiden Ländem wurde im Anschluß an die Unabhängigkeitserklärung die Schaffung einer neuen Verfassung beschlossen; in beiden Ländern lagen die ersten diskussionsf ähigen Entwürfe im Sommer 1991 vor. Seit dieser Zeit wird sowohl in Weißrußland wie auch in der Ukraine an den Verfassungsentwürfen herumgebastelt, wobei die politischen Problempunkte nur zum Teil identisch sind. Problematisch ist in beiden Lăndem analog dem Beispiel der Russsischen Föderation das Verhälmis zwischen Präsident und Parlament; äußerst problematisch ist auch in beiden Ländem die wirtschaftliche Situation, dieses ebenso wegen der Beharnungskraft der alten Machtstrukturen wie auch wegen des Desinteresses aus westlichen Wirtschaftskreisen. Ein spezifisch ukrainisches Problem ist darüber hinaus noch die Frage "Föderalismus / Einheitsstaat": Die starken nationalistischen Strömungen sind aus Furcht vor zu großer Autonomie oder gar Abspaltung der Krim strikt gegen eine föderale Struktur des Staates; gerade diese rigide Haltung könnte doch die zentrifugalen Tendenzen verstärken. Dazu kommt ein generelles Desinteresse der Parlamentsabgeordneten an einer neuen Verfassung: In beiden Ländern wurden die Parlamente nach dem von Gorbacev in der Union erstmals eingeführten, zwar endlich freien aber noch ungleichen Wahlsystem gewählt. Folglich sind unter den Abgeordneten die Kommunisten und ihnen nahestehende Richtungen überrepräsentiert. Eine neue Verfassung würde automatisch die Forderung nach Neuwahlen auf den Tisch bringen, um dem unter der neuen Verfassung agierenden Parlament auch eine echte demokratische Legitimation zu geben; an diesem Statusverlust haben die Abgeordneten verständlicherweise kein Interesse. Daher behilft man sich vorläufig mit einem regelmäßigen "updating" der alten Verfassungen etwa im Bereich der Präsidentschaft oder auch der Erschaffung von Verfassungsgerichten. Parallel dazu wird jeweils die Absicht bekräftigt, eine neue Verfassung zu beschließen, ohne daß es jemals dazu kommen

3 Siehe die Veröffentlichung des Entwurfs in der Kasachskaja Pravda v. 2.2.1993; siehe die Verfassung Usbekistans in der Taskentskaja Pravda v. 15.12.1992. Die estnische Verfassung wurde am 28.6.1992 per Referendum beschlossen; siehe die separate Veröffentlichung, Tallin, 1992. 
würde. ${ }^{4}$ Im Falle Estlands war der VerfassungsgebungsprozeB unproblematisch: Die Angst vor der übermächtigen Russischen Föderation und der fast schon verzweifelte Versuch einer möglichst schnellen nationalen Identitätsfindung führten hier zu einer schnellen Verabschiedung der Verfassung.

Den ProzeB der Verfassungsgebung in der Russischen Föderation kennzeichnet ein dramatisches Hin und Her, eine völlig unüberschaubare Anzahl von Akteuren, undurchsichtige Winkelzüge und ein zumindest zum Teil tragisches Ende. Wir wollen den Ablauf der Geschehnisse etwas genauer betrachten. Es begann, ähnlich wie in WeiBruBland und in der Ukraine, mit einem Entwurf des Obersten Sowjets bzw. des Volksdeputiertenkongresses der damaligen RSFSR: Auch hier wurde die Verfassungskommission zur Schaffung der neuen Verfasssung im Sommer 1990 (in Abgrenzung gegen die UdSSR) zusammengerufen. Seit 1991 lag ein Entwurf vor, der ständig überarbeitet und verbessert (oder auch verschlechtert) wurde und der im VolksdeputierenkongreB das Schicksal der Verfassungsentwürfe von WeißruBland und der Ukraine teilte: Immer wieder sollte der Entwurf beraten werden, immer wieder kam es zu zum Teil ganz erheblichen Änderungen der noch aus sozialistischer Zeit stammenden alten Verfassung und zu einem Vertagen des Projekts einer neuen Verfassung. Immerhin aber billigte der VolksdeputiertenkongreB im April 1992 im Grundsatz und immerhin wurde das Projekt vom Obersten Sowjet - freilich hatte dieser nicht die Kompetenz der Verfassungsgebung - artikelweise beraten. 5

Dieser Verfassungsentwurf der Russischen Föderation ist mit dem Namen des Sekretärs der Verfassungskommission, O.G. Rumjancev, verbunden; es war zunächst der einzige Entwurf. Im Frühjahr 1992 erschien ein alternativer, der Rechtswissenschaft bzw. den Reformern zuzuordnender Verfassungsentwurf, für den der Bürgermeister von Leningrad, A.A. Sobcak, und der (freilich nicht als Reformer verschrieene) "Papst" der sowjetischen Staatsrechtswissenschaft, S.S. Alexejev, verantwortlich zeichneten. Dieser Entwurf, stark von der französischen Rechtstradition geprägt, brachte die Verfassungskommission von Rumjancev unter einen ganz erheblichen Legitimationsdruck.6 Das Verwirrspiel sollte sich freilich noch komplizierter gestalten: Im Vorfeld der im April 1992 anstehenden Sitzungsperiode des Volksdeputiertenkongresses verdichteten sich die Gerüchte, Jelzin (der pikanterweise

4 Siehe die regelmäßigen Berichte im vom Center for the Study of Constitutionalism in Eastem Europe der Law-School der University of Chicago herausgegebenen East European Constitutional Review, jeweils und dem Titel "Constitution Watch".

5 Siehe die ausführliche Dokumentation des (Versuch eines) Verfassungsgebungsprozesses in dem von der Verfassungskommission herausgegebenen "Konstitucionnyj vestnik", dessen Erscheinen am 31.8. (Verfassungskommission) bzw. am 12.9.1990 (Vorsitzender des Obersten Sow jets) beschlossen wurde und von dem 13 Folgen mit Materialien zum Prozeß der Verfassungsgebung erschienen sind.

6 Siehe die Veröffentlichungen der Parlamentszeitung "Föderation" 1992, Nr. 14/15, 18 ff., sowie die spätere Veröffentlichung durch die Presseagentur "Novosti" 1992. 
Vorsitzender der Verfassungskommission des Obersten Sowjets war) werde einen eigenen Entwurf veröffentlichen. Die Dementis waren ebenso heftig wie die Wirkung, die der dann Anfang April veröffentlichte Verfassungsentwurf - nach seinem Autor als sogenannter Sachraj-Entwurf bezeichnet - verursachte. 7

Auf die Praxis der Verfassungsgebung hatte freilich dieser Ubberfluß an Verfassungsentwürfen keine Auswirkung: Man verbesserte weiter die existierende Verfassung, z.B. durch die Einfügung eines neuen Grundrechtskapitels. 8 Bewegung kam in die Verfassungsgebung erst wieder im Dezember 1992: Sie wurde zu einem der Schlachtfelder, auf dem der Kampf um die Macht zwischen dem Parlament und dem Präsidenten ausgetragen wurde. Die Entwicklung soll im einzelnen hier nicht dargestellt werden:9 In der Konsequenz der völligen Zerrüttung und auf der Woge der im Referendum vom April 1992 erworbenen neuen Legitimität entschloß sich Jelzin, am Volksdeputiertenkongreß vorbei eine neue Verfassung durch eine eigene verfassungsgebende Versammlung beraten zu lassen. Zur politischen Durchsetzung schloß er dabei eine ziemlich unheilige Allianz mit den Republiken und Gebieten, die sich als Belohnung eine größere Autonomie unter der neuen Verfassung versprachen. Der dann veröffentlichte Entwurf stellte eine von S.S. Alexejev überarbeitete Fassung des Sachraj-Entwurfs dar. 10 Der Oberste Sow jet und der Volksdeputiertenkongreß reagierten schnell: Als kleineres Úbel zog man den eigenen Verfassungsentwurf unter dem Ladentisch hervor und veröffentlichte diesen, zusammen mit einem detaillierten Zeitplan, der die Verabschiedung im Herbst bzw. im Winter 1993 vorsah.11

Es kam dann während des Sommers zu einer Ubberarbeitung des Jelzinschen Verfassungsentwurfs durch die von ihm einberufene Verfassungsversammlung; in dieser Versammlung waren Jelzin-Anhänger, Vertreter der Republiken und Gebiete wie aber auch Mitglieder des

7 Föderation, 1992, Nr. 16, $17 \mathrm{ff}$.

8 Seit 1990 hatte es acht Verfassungsänderungen gegeben, mit denen ganz wesentliche Änderungen (Präsidentschaft, Verfassungsgericht, Grundrechtskapitel, Föderationsvertrag) in die Verfassung eingef ügt wurden, am 31.5.1990, 16.6.1990, 15.12.1990, 24.5.1991, 1.11.1991, 21.4.1992, 9.12.1992 und 10.12.1992

9 Siehe dazu meine etwas eingehendere Darstellung in "Präsident, Parlament(spräsident) und ein sichtbarer Dritter. Wohin verschwand die Macht in der Russischen Föderation, in: Staatswissenschaf ten und Staatspraxis, 1993, $139 \mathrm{ff}$.; siehe im übrigen die Berichte in East European Constitutional Review 1992, Nr. 2, 6 ff.; 1992, Nr. 3, 9 ff.; 1993, Nr. 1, 10 ff.; 1993, Nr. 2, 15 ff.; 1993, Nr. $3,20 \mathrm{ff}$.

10 Siehe die Zusammenstellung aller zentralen Akte (sowie des Verfassungsentwurfs in der Fassung vom 12.7.1992) in: Konstitucionnoe sovescanie, 1993, Nr. 1, etwa mit der personellen Zusammensetzung der Arbeitsgruppe, (9 ff.); siehe auch den langen Arikel von S.S. Alekseev zu "seiner" Verf assung in der "Nezavisimaja Gazeta" (NG) vom 25.5.1993

11 Siehe den Beschluß des Obersten Sowjets (OS) v. 29.4.1993, Nr. 4907-1, Rossiskaja Gazeta (RG) v. 8.5.1993, auch veröffentlicht zusammen mit dem Projekt, einer Stellungnahme zum Projekt des Präsidenten sowie sonstigen Materialien in dem Band: Das Projekt der Verfassung der Russischen Föderation, 1993 (russ.), hrsg. vom OS der Russischen Föderation. 
Obersten Sowjets - pro und contra Jelzin - sowie Angehörige anderer zentraler Institutionen der Russischen Föderation vertreten. 12 Als Abschluß ihrer Beratungen legte die Versammlung - nach einigen saftigen Skandalen - eine Zwischenversion der Verfassung am 12.7.1993 vor, in der Sache waren hier das Verfassungsprojekt des Obersten Sowjets und das Jelzinsche Projekt miteinander verschmolzen worden. Dieser Entwurf sollte entsprechend den Planungen im September 1993 weiter überarbeitet und zu einer endgültigen Beschlußvorlage vervollkommnet werden; der Modus der Verabschiedung war, angesichts der geringen Chancen im Volksdeputiertenkongreß, nicht klar.13 Die Entwicklung verlief dann jedoch ganz anders: Angesichts der hohen Wahrscheinlichkeit einer weiteren Beschneidung der präsidialen Kompetenzen in der kommenden Sitzungsperiode des Volksdeputiertenkongresses (ganz zu schweigen von eventuellen weiteren impeachment-Versuchen) löste Jelzin den Obersten Sowjet auf. Der Rest der traurigen Ereignisse ist Zeitgeschichte; es sei unterstrichen, daß schon vor Ausbruch der gewalttätigen Auseinandersetzungen beide Seiten und leider auch das russische Verfassungsgericht in guter sowjetischrussischer Tradition sich nicht einmal mehr um den Anschein der Verfassungs-/ Gesetzestreue bemühten. 14 Nach der Stürmung des Parlaments führte Jelzin mit einem Bündel von

12 Die begleitende Presse war und ist äußerst umf angreich, sowohl von joumalistischer und rechtswissenschaftlicher Seite wie auch aus der antipräsidialen "Fraktion", siehe etwa RG v. 15.6.1993 ("Die Verfassungsversammlung als Instrument des politischen Kampfes"); NG v. 13.5.1993 ("Die Regionen entscheiden das Schicksal des Grundgesetzes"); NG v. 15.5.1993 ("Der Verfassungsprozeß schreitet voran"); NG v. 27.5.1993 ("Verfassungsultimatum an den Präsidenten"); NG v. 22.5.1993 ("Schritt für Schritt erobent der Präsident das Verfassungsfeld"); von den Beteiligten siehe etwa die Artikel von R.I. Chasbulatov, Ist eine Balkanisierung Rußlands möglich?, RG v. 27.5.1993; O.G. Rumjancev / V. Lafickij, Zwei Projekte - zwei Ansichten über Gewaltenteilung; dieslb., Der Föderalismus in den zwei Verfassungsprojekten, NG v. 1.6.1993. Siehe auch die sehr kritischen Äußerungen seitens des (damaligen)Verfassungsgerichtspräsidenten VD. Zor'kin: Ein Gesetz, das auf verfassungswidrigem Feld angelegt ist, führt nicht zum nationalen Konsens, RG v. 18.5.1993; V. Sobakin (Mitarbeiter des Gerichts und ehernaliges Mitglied des Komitees für Verfassungsauf sicht): Über die Menschenrechte in den Verfassungsprojekten, NG v. 28.5.1993. - Zu den Querelen um das Gericht und seine Gespaltenheit zwischen der Mehrheit um Zor'kin und der propräsidialen Minderheit siehe etwa in: NG v. 16.6.1993; RG v. 15.6.1993.

13 Siehe etwa den Beschluß des OS v. 4.6.1993 in: KS 1993, Nr.1, 63 ff.; siehe auch ebenda die unterschiedlichen Vorstellungen zur Verabschiedung der Verfassung ( $94 \mathrm{ff}$. sowie $155 \mathrm{ff}$. das Projekt eines "Gesetzes über die Verabschiedung der Verfassung").

14 Siehe den grundlegenden Ukaz Nr. 1400 zur Parlamentsauflösung v. 21.9.1993, Izvestija (Izv) v. 23.9.1993, sowie dann, nach dem Sturm des Parlaments, den Ukaz v. 7.10.1993 über die Rechtssetzungstätigkeit in der Zeit der "etappenweisen Verfassungsreform in der RF" (so die Bezeichnung der Zeit bis zur Neuwahl des Parlaments nach der Auflösung des alten Parlaments, RG v. 9.10.1993, S. 1. Der Ukaz Nr. 1400 wurde in einer Nacht- und Nebelentscheidung des Verfassungsgerichts v. 21.9.1993 für verfassungswidrig erklärt, s. RG v. 23.9.1993, S. 2; der Antrag des Parlaments auf Verfassungswidrigerklärung des Ukaz kam erst am 22.9.1993 zum Verfassungsgericht, siehe RG v. 23.9.1993. Zur Entscheidung des Verfassungsgerichts gab es zwei Sondervoten von Ametistov und Morscakova, die nicht veröffentlicht wurden. Auch die vom Parlament am 22.9. ausgesprochene Amtsenthebung des Präsidenten folgte nicht ganz dem vorgeschriebenen 
Maßnahmen eine sehr monistische Präsidialherrschaft ein und zog die föderalen Schrauben wieder zentralistisch fest an: Ein nochmals überarbeiteter und nicht überraschend sehr präsidentenfreundlicher Verfassungsentwurf wurde dann der Öfentlichkeit vorgestellt. 15 Am 12.12.1993 wurde die Verfassung per Referendum beschlossen; die gleichzeitig stattfindenden Wahlen und ihre Ergebnisse demonstrierten mit unerfreulicher, aber notwendiger Klarheit die Gespaltenheit, Verwirntheit und Radikalität der russischen Gesellschaft.16

Der Stand der Verfassungsgebung bzw. der verabschiedeteten Verfassung in den betrachteten Ländem mag also mit emst, aber nicht hof fnungsvoll charakterisiert werden. Interessant waren die Verfassungen nur dort, wo sie als Trumpfkarten im Kartenspiel um die Neuverteilung der Macht, sei es innerhalb des Landes, sei es auch gegenüber dem russisch-sowjetischen Zentrum eingebracht werden konnten. Eine darüber hinausgehende Identifizierung mit dem Projekt "Verfassungsstaat" scheint es in den Eliten nicht zu geben. Man muß freilich ehrlicherweise hinzufügen, daB auch in der Bevölkerung das Interesse an neuen Verfassungen sich in Grenzen hält: Man hat dort andere Sorgen und kann sich nicht vorstellen, $\mathrm{da} ß$ und wie alte oder neue Verfassungen sich auf das Leben des Einzelnen positiv auswirken kömnten. Wie sollte man auch: In den historischen Gedächtnissen dieser Gesellschaften sind funktionierende Verfassungen als schöne Erlebnisse nicht enthalten.

\section{B. Inhalte, Gemeinsamkeiten und Unterschiede}

Da es zu den Besonderheiten der von uns betrachteten Länder gehört, gerade im Zustand der Hoffnungslosigkeit ganz überraschende Dinge zu vollbringen, wollen wir nichtsdestotrotz einen eingehenden Blick auf die Verfassungen bzw. die Verfassungsprojekte werfen,

Weg: Nach Art. 121/10 alte Verfassung Russische Föderation (aVerRF) war hierzu eine Stellungnahme des Verfassungsgerichts bezüglich der Verfassungswidrigkeit der Handlung des Präsidenten erforderlich. Das Verfassungsgericht hatte ohne Antrag seine Entscheidung vom 21.9.1993 als ein solches Gutachten eingeordnet; wiewohl die Stellungnahme ein Verfahren ist, bei dem das Gericht das Recht der Eigeninitiative hat, Art. 74 Abs. 2 VerfGGRF, ist fraglich, ob das gewählte Verfahren dem Gesetz über das Verfassungsgericht entsprach. Nicht weniger verfassungswidrig waren die Handlungsweisen des OSs: Am 22.9.1993 entschied dieser mit Beschluß Nr. 5780-I über die Beendigung der Machtbefugnisse des Präsidenten (die Kompetenz hätte gem. Art. 121/10 Verf. nur der Volksdeputiertenkongreß gehabt; eine Entscheidung dieses Typs gibt es überhaupt nicht) sowie mit Beschluß 5783-I über den Antrag an das Verfassungsgericht gegen den Ukaz Nr. 1400, siehe RG v. 23.9.1993.

15 Siehe RG v. 10.11.1993; vgl. auch die englische Übersetzung des Europarats im Dokumentationsanhang in diesem Heft.

16 Auch die Partei von Zirinovskij hat ein eigenes Verfassungsprojekt vorgelegt, daß freilich nur 29 Artikel hat (erschienen im Eigenverlag der Partei). Zirinovskij war im übrigen auch Teilnehmer der Verfassungsgebenden Versammlung und sprach sich dort für die Bewahrung nationaler Eigenheiten und ein starkes Präsidialregime aus: siehe KS, Nr. 1, 50 ff.; siehe im übrigen als weiteren Entwurf noch den Entwurf der Kommunisten: RG v. 24.7.1993. 
dies unter zwei Aspekten: Zunächst wollen wir schlicht die Gemeinsamkeiten und Unterschiede der Verfassungstexte betrachten, sowohl untereinander wie auch im Verhältnis zu anderen, westlichen Verfassungstexten. Sodann wollen wir uns fragen, ob gewisse Gemeinsamkeiten und Gesetzmäßigkeiten in allen Texten feststellbar sind, die Grundlage für die Konstatierung einer (post?)sozialistischen Kultur der Verf assungsgebung sein könnten.

\section{Der Aufbau der Verfassungen}

Vom Aufbau her folgen die meisten Verfassungsentwürfe einem einheitlichen Muster. Nach einer regelmäßig sehr blumigen Präambel enthält der erste Abschnitt die Staatsstrukturbestimmungen und eine Standortbestimmung des neuen Staatswesens in der Völker- und Völkerrechtsgemeinschaft. Es folgt regelmäßig das Kapitel über die Grundrechte, eingeleitet von allgemeinen Bestimmungen wie der Vorstaatlichkeit der Grundrechte, der Rechtssubjektivität der Bürger usw. Der Katalog der konkreten Grundrechte beginnt meistens mit dem Gleicheitssatz und normiert dann in der Folge zunächst die einzelnen Abwehrrechte, dann regelmäßig einen sehr ausführlichen Katalog von sozialen Leistungsrechten und schließlich einen meist auch ausführlichen Katalog von allgemeinen Grundpflichten. Erwähnenswert ist weiterhin, daß die Verfassungsentwürfe Rußlands (des Obersten Sowjets), der Ukraine und auch Kasachstans nach den Grundrechten in Form von Abwehrund Leistungsrechten in einem weiteren Abschnitt das Thema "Gesellschaft" regeln.17 Dieses Kapitel würde in unserer Terminologie die objektiv-rechtliche Seite der Grundrechte festschreiben. In den unterschiedlichen Verfassungstexten der Russischen Föderation folgt als nächstes das Kapitel über die Bundesstaatlichkeit, wobei sowohl die alte wie auch die jetzt geltende Verfassung hierbei den zwischen den Subjekten der Föderation geschlossenen Föderationsvertrag als Kapitel in die Verfassung integrieren.18 Die anderen Staaten, die keine Bundessstaaten sind, bzw. keine Bundesstaaten sein wollen, regeln die Frage der territorialen Aufgliederung regelmäßig im vorletzten Teil der Verfassung zusammen mit der kommunalen Selbstverwaltung. 19

17 Art. 57 ff. Verfassungsentwurf Russische Föderation (VerfERF), OS; Art. 67 ff., Verfassungsentwurf Ukraine (VerfUkr); siehe auch Art. 45 ff., Verfassung Kasachstan (VerfKas), Kap. "Die Gesellschaft, die Grundlagen ihrer Struktur".

18 Siehe Art. 65 ff. neue Verfassung Russische Föderation (nVerfRF); Art. 68 ff. aVerfRF; der Entwurf des OSs enthielt keine Bestimmungen zur Föderation, dafür aber den Föderationsvertrag als Anlage zur Verfassung, siehe "Das Verfassungsprojekt der Russischen Föderation", 56 ff.

19 Siehe etwa Art. 120 ff. Verfassungsentwurf Weißrußland (VerfEWeißr); Art. 154 ff. Verfassung Estland (VerfEst); Art. 89 ff. Verfassung Kasachstan (VerfKas); anders Art. 104 ff./Art. 195 ff. VerfEUkr: Die Ukraine ist gernäß diesen Vorschriften ein Einheitsstaat mit einer autonomen Republik, wobei die rechtliche Situation der Republik Krim im Abschnitt vor dem politischen System geregelt ist, die sonstige Aufteilung danach. Ähnlich auch Art. 68 ff. Verfassung Usbekistan (VerfUs), die neben den üblichen administrativen Aufteilungen noch die Republik Karakal- 
Es folgt als Zentralteil die Regelung des politischen Systems, üblicherweise in der Anordnung Parlamente / Organe des Parlaments / Präsident / Regierung. Nicht überraschend durchbricht die neue Verfassung der Russischen Föderation diese Anordnung: Das erste Kapitel ist hier die Regelung der Präsidentschaft; es folgh, als Zuckerstuckchen für die Regionen und Republiken, die Regelung der zweiten, föderalen Kammer des Parlaments, und erst dann findet man die Regelung für die erste Kammer.20 Ein wenig heimatlos sind das Wahlsystem und die Grundrechte des Wahlrechts, die manchmal bei den Staatsstrukturbestimmungen, manchmal im Grundrechtskapitel und manchmal im Abschnitt über das Parlament zu finden sind.21 Den AbschluB der Regelung des politischen Systems bildet regelmäßig die Gerichtsbarkeit, teilweise mit Einschluß der Verfassungsgerichtsbarkeit; in anderen Texten ist der Verfassungsgerichtsbarkeit ein eigenes Kapitel am Ende des Verfassungsprojekts oder der Verfassung gewidmet.22 Es folgen einheitlich die Kapitel über das Finanzsystem, die kommunale Selbstverwaltung und die Änderung der Verfassung; die

pakstan anerkennt und dieser sogar in Ar. 74 das Recht zam Austritt aus der Republik Usbekistan gibt (auf der Grundlage eines Referendums).

20 Siehe etwa in der Reihenfolge Parlament / Präsident / Regierung die Ar. 62 ff., 75 ff., 84 ff. VerfKas; Art. 80 ff, 93 ff, 100 ff. VerfEWeiBr, siehe demgegenüber Art. 80 ff. / Präsident (Entwurf April: Ar. 77 ff.; Entwurf Juli: 80 ff.); Ar. 94 ff. / Parlament (Entwurf April: Ar. 89 ff.; Entwurf Juli: 93 ff.); Art. 110 ff. / Regierung (Entwurf April: Ar. 106 ff.; Entwurf Juli: 110 ff.) nVerfR; der Entwurf des Obersten Sowjet regelte in der normalen obigen Reihenfolge in den Artikeln 85 ff., 93 ff., 98 ff. Auch eine bewuBe "abweichlerische" Verfassung wie die estnische folgt diesem Regelungsmuster, siche Ar. 59 ff., 77 ff., 86 ff.

21 Siehe Art. 3 Abs. 2 u. 3, 32 (Wahlrecht als Grundrecht), Ar. 81 (Präsident), Art. 96 (Parlament) nVerfR; nur beim Präsidenten sind die Wahlrechtsgrundsätze allgemein, gleich, direkt und geheim geregelt; das Prinzip der Freiheit der Wahl ist pikanterweise ausdrücklich nirgendwo festgeschrieben. Siehe weiter die Ar. 109 ff. VerfKas, wiederum ohne den Grundsatz der freien Wahl; Ar. 32 ff., 117 VerfUs, wiederum ohne den Grundsatz der freien Wahl; Art. 64 ff. VerfEWeißr, im Anschluß an die Grundrechte; hier ist der Grundsatz der Freiheit der Wahl normiert als positive und negative Wahlrechtsfreiheit; siehe weiter Art. 118 ff. VerfEUkr (Abschnitt: Die Staatsmacht); Art. 120 enthält sämtliche W ahlrechtsgrundsätze, auch in Nr. 5 das Prinzip der Freiheit der Wahl; die Bestimmungen zur Wahl folgen aber bezeichnenderweise den Bestimmungen über das Referendum. Siehe weiter Ar. 57 VerfEst (vor der Regelung der Staatsorgane); hier wird jedoch nur das Wahlrecht als solches eingeräumt.

22 Die Verfassungsgerichtsbarkeit ist in einem eigenen Kapitel geregelt, in Ar. 128 ff. VerfEWeißr; Ar. 95 Abs. 1 u. 2, 130, 131 VerfKas; Art. 203, 204, 239 ff. VerfEUkr; Kasachstan und die Ukraine ordnen die Verfassungsgerichte, nach Erwähnung im Gerichtsteil, im Abschnitt über den Schutz der Verfassung ein. - Im Kapitel über die Gerichtsbarkeit regeln die Ar. 125 ff. nVerfRF; ebenso Art. 101 VerfERF/OS sowie Art. 108 ff. VerfUs. Die Verfassung Estlands folgt in Art 149 Abs. 3 Nr. 2 dem amerikanischen Supreme-Court-Modell und hat kein eigenes Verfassungsgericht. Einige der Verfassungsentwürfe bzw. Verf assungen enthalten weniger eine Regelung der Verfassungsgerichtsbarkeit als vielmehr eine verf assungsrechtliche Eröffnung dieser Art von Gerichtsbarkeit mit der Möglichkeit des Gesetzgebers, das Verfassungsgericht im einzelnen zu regeln. 
Problematik der Fortgeltung alten Rechts wird teilweise in der Verfassung, teilweise in einem Ubergangsgesetz geregelt. 23

\section{Die Staatsstrukturprinzipien}

Bei den Staatsstrukturprinzipien folgen alle Verfassungen einem Grundmodell mit Variationen im einzelnen. Durchgängig werden die Prinzipien Demokratie, Rechtsstaat, Gewaltenteilung und Sozialstaat festgelegt. In allen Verfassungen findet sich auch ein Artikel, der das Wohlergehen des Individuums und die Verwirklichung von dessen Rechten zum höchsten Staatsziel erhebt.24 Darüber hinaus gibt es eine Reihe von Vorschriften, die Ausdruck der unsicheren Identität dieser plötzlich neu entstandenen Staaten sind: So wird die Volkssouveränität und vor allem die Souverănität gegenüber anderen Staaten überall stark hervorgehoben, ebenso die Unverletzlichkeit und Unveränderlichkeit des Territoriums;25 zum Teil finden sich sogar Vorschriften, nach denen jede Mitgliedschaft in (nicht näher definierten) internationalen Vereinigungen verfassungswidrig wäre, der Zusammenschluß zu solchen Vereinigungen also eine Verfassungsänderung notwendig machen würde.26 Ein wenig kontrafaktisch wird in allen Verfassungen (in Rußland ist hier die Lage anders) eine Staatssprache festgelegt.27 Kontrafaktisch sind diese Festschreibungen, weil große Bevölkenungsteile, ja zum Teil mehr als die Hälfte, diese Staatssprache schlecht oder gar nicht

23 In der Verfassung regelt dies die neue Verfassung der Russischen Föderation (Abschnitt II), das Verfassungsprojekt der Russischen Föderation des Obersten Sowjet (3. Teil) sowie die Verfassung Kasachstans (V. Abschnitt). Eigene Gesetze zeigt der Verfassungsentwurf Weißrußlands, die Verfassung Estlands sowie die Verfassung Usbekistans; unklar ist die Einordnung der Übergangsbestimmungen in dem Verfassungsentwurf der Ukraine.

24 Art. 1, 2 (Individuum), 6 (Sozialstaat) nVerfRF; Ar. 1 Abs. 1, 2 (Individuum) VerfERF/OS; I Abs. 2, III (Individuum), IV (Gewaltenteilung) GrundlagenVerfKas; es fehlt eine Regelung der Sozialstaatlichkeit. (Bei den "GrundlagenVerfKas handelt es sich um das Eingangskapitel der Verfassung, das gem. Ar. 129 Abs. 2 VerfKas besonders durch das Erfordemis einer Mehrheit von 3/4 vor Verfassungsänderungen geschützt ist.) Sehr unklar ist die Verfassung Usbekistans mit Art. 1 / Demokratie, Art. 11 /Gewaltenteilung, Ar. 14 / sozialer Gerechtigkeit und Gesetzlichkeit; windelweich Art. 1, 3, 4 VerfEst - es fehlt das "Wohlergehen des Individuums" und die Sozialstaatlichkeit; Art. 1 Abs. 1 u. 2 (Individuum) VerfEWeißr; Art. 1, 2 VerfEUkr.

25 Ar. 2 VerfEst; Art. 1 Abs. 2, 9 VerfEWeißr; Ar. 7 VerfEUkr; Ar. 3 Abs. 2 VerfUs; II GrundlagenverfKas; Art. 4, 5 nVerfRF - spezifisch gegen die zentrifugalen Kräfte gerichtet, während die anderen Staaten sich eher gegen äußere Bedrohung (nämlich die Russische Föderation) richten; siehe weiter Art. 1 Abs. 2 VerfERF/OS.

26 Siehe etwa Art. 8 Abs. 2 i.V.m. Ar. 1 Abs. 3 VerfEWeißr; tendenziell auch Ar. 123 VerfEst.

27 Art. 6 VerfEst; VII Grundlagen VerfKas; Ar. 17 VerfEWeißr; der Verfassungsentwurf der Ukraine regelt die Staatssprache nicht, sondem gibt der Nationalversammlung die Kompetenz, den "Status der Sprachen" zu bestimmen, Ar. 135 Nr. 5 (die Kompetenz zur Regelung der Rechte der Minderheiten findet sich in Nr. 4, deren Rechte in Ar. 13, dem Recht auf das nationale Erbe); Ar. 4 VerfUs; Art. 67 nVerfRF (Kap. Föderalismus zu Beginn); Art. 84 VerERF/OS (am Ende des Kapitels zum Föderalismus). 
beherrschen. Ich nehme an, daß man mit diesen Artikeln schlafende Hunde wecken wird. Bemerkenswert ist weiter die Festschreibung einiger Staaten als laizistisch bzw. auch das Fehlen einer solchen Bestimmung: So enthält etwa die Verfassung von Kasachstan oder auch der neuen slawischen Staaten diese Festlegung, während eine ähnliche Bestimmung bei der usbekischen Verfassung fehlt.28

Bekarmtlich ist jede Verfassung eine Antwort auf die Vergangenheit, und auch die Staatsstrukturbestimmungen der von uns betrachteten Verfassungen und Verfassungsentwürfe enthalten solche Antworten. Durchgängig findet sich überall das Verbot der Ideologisierung des Staates und der Grundsatz, daB keine gesellschaftliche Gruppe sich den Staat aneignen dürfe.29 Betont wird auch überall die Bindung der Staatsgewalt an Gesetz und Verfassung und vor allem die direkte Wirkung der Verfassung und der Gesetze. 30 Darüber hinaus enthalten alle Verfassungen besondere Hervorhebungen der Völkerrechtsfreundlichkeit des Rechtssystems: Den allgemein anerkannten Regeln des Völkerrechts bzw. den ratifizierten Menschenrechtspakten wird gegenüber dem innerstaatlichen allgemeinen Gesetzesrecht der Vorrang eingeräumt, womit wie im Grundgesetz eine weitere Stufe in der Normenhierarchie zwischen formellem Gesetz und Verfassung begründet wird.31 Schließlich weisen die meisten Verfassungen eine Bestimmung auf, wonach Rechtsnormen nur dann verbindlich sein können, wenn sie veröffentlicht worden sind:32 Zum Teil wird dies dahin abgeschwächt, daß dies nur insoweit gilt, wie durch die Rechtsnorm Freiheiten und

28 Art. 14 nVerfRF; abgemilder in Ar. 6 VerfEUkr; sehr unklar auch Ar. 4, 16 VerfEWeißr; siehe demgegenüber Art. 40 Abs. 2 Satz 2 VerfEst (im Grundrechtskapitel); ideologisch neutral Ar. 12 VerfUs (ohne Erwähnung der Religion); signifikant demgegenüber Art. 58 VerfKas, bes. Art. 58 Abs. 2: Verbot politischer Parteien auf religiöser Grundlage (Kap. "Die Gesellschaft: Grundlagen ihrer Struktur").

29 V Abs. 2 GrundlagenVerfKas; Art. 7 Abs 2 Satz 2 VerfUs; ähnlich auch das Verbor, die Republik Usbekistan zu vertreten, Art. 10 Abs. 2; Art. 3 Abs. 4 VerfEUkr; siehe auch Art. 6 Abs. 4, 3 VerfEWeißr; in der estnischen Verf assung fehlt, auch im Gundrechtsteil, eine entsprechende Vorschrift; siehe weiter Art. 13, besonders Abs. 2, 4 nVerfRF; Art. 4 Abs. 3 VerfERF/OS.

30 Art. 15 nVerfRF; Art. 3 Abs. 2 VerfERF/OS; VII GrundlagenVerfKas; Ar. 5 Abs. 2 VerfEUkr; Art. 7 VerfEWeißr; Art. 15 VErfUs; Ar. 102 VerfEst.

31 Präambel Verfassung Usbekistan; im zentralen Art. 17 der Verfassung fehlt freilich eine entsprechende Konkretisierung; Ar. 3 VerfKas; Art. 8 Abs. 1 VerfEWeißr; Ar. 123 Abs. 2 VerfEst (Höherrangigkeit ratifizienter intemationaler Vertäge); Ar. 15 Abs. 4 nVerfRF; siehe auch die Wiederholung bei den Vorschriften über Minderheiten in Ar. 69 nVerRF; Ar. 3 Abs. 4 VerfERF/OS, ebenfalls beschränkt auf ratifiziente Verträge; relativ reservien Art. 8 Abs. 1 VerfEUkr ("achtet").

32 Art. 3 Abs. 2 VerfEst; Art. 15 Abs. 3 nVerfRF; Ar. 3 Abs. 3 VerfERF/OS; Art. 7 Abs. 3 VerfWeißr; keine entsprechende Bestimmung im Grundlagenkapitel enthalten die Verfassungen von Kasachstan und Usbekistan sowie der Verfassungsentwurf der Ukraine. 
Rechte des Bürgers berührt oder eingeschränkt werden. Schließlich regeln alle Verfassungen die Staatssymbole und die Hauptstadt. 33

\section{Die Grundrechte}

Alle Verfasssungen erhalten einen ausführlichen Grundrechtskatalog, wobei regelmäßig ein erster Teil gewisse allgemeine Fragen, ein zweiter Teil die Abwehrrechte und ein dritter Teil die Leistungsrechte des Individuums regelt; den Abschluß bilden durchgängig die Grundpflichten des Bürgers. Bei den allgemeinen Fragen werden zum einen Fragen wie Staatsbürgerschaft, Schutzpflicht des Staates gegenüber seinen Bürgern, Ausländergrundrechte und ähnliches angesprochen;34 zum anderen befindet sich hier regelmäßig eine allgemeine Schrankenregelung, die normalerweise in formeller Hinsicht das Prinzip des Gesetzesvorbehalts in Form des Parlamentsvorbehalts anordnet und in inhaltlicher Hinsicht die Schranken der Grundrechte in der äußeren Sicherheit, in der öffentlichen Ordnung, dem Schutz der Moral und der Gesundheit der Bevölkerung sowie den Rechten und Freiheiten der Bürger sieht.35 Die Rechte im einzelnen ähneln einerseits dem Grundrechtskatalog etwa des Grundgesetzes oder anderer modemer westlicher Verfassungen: Leben, Gesundheit, Glaubensfreiheit, Meinungs- und Versammlungsfreiheit, Vereinigungsfreiheit, die wirtschaftlichen Grundrechte, Asylrecht und vor allem auch die Justizgrundrechte wie etwa das Verbot der Rückwirkung von Gesetzen, die Unschuldsvermutung und ähnliches finden sich allerorten.36 Daneben gibt es freilich einige Besonderheiten, die in westlichen Verf as-

33 Ar. 250 ff. VerfEUkr; IX GrundlagenVerfKas; Ar. 5, 6 VerfUs; Art. 7 VerfEst; Art. 70 nVerfRF (Kap. "Föderalismus"); Art. 128, 129 VerfERF/OS (Schlußvorschriften); Art. 19, 20 VerfEWeißr.

34 Ar. 18 nVerfRF: direkte und programmierende Wirkung der Grundrechte für alle Staatsgewalten (in der vorherigen Version hieß es "verpflichtet"); viel ausführlicher die Ar. 13 bis 19 VerfERF/OS wie auch die Art. 31 bis 37 der aVerfRF. Die Frage der (Grundrechts?)Mündigkeit regelt Ar. 60 nVerfRF; Doppelstaatsbürgerschaft und Ausländerrechte regeln die Ar. 62 u. 63. Siehe weiter Art. 4 ff. VerfKas; Ar. 21 bis 23 VerfUs; Ar. 11 ff., 15 ff. VerfEUkr; anders der Verfassungsentwurf Weißrußlands, der die Staatsbürgerschaftsfragen im Kapitel "Grundlagen" (Ar. 10) vor den Grundrechten regelt. Die Verfassung Estlands regelt zumindest in Ar. 8, 9 die ganze zentrale Frage der Staatsbürgerschaft und der Ausländerrechte.

35 Ar. 17 Abs. 3 nVerfRF; siehe dazu noch den allgemeinen qualifizierten Parlamentsvorbehalt in Ar. 55 Abs. 3; der Sache nach gleich Ar. 13 Abs. 4 VerfERF/OS; siehe weiter Ar. 2 VerfKas; sehr knapp Art. 20 VerfUs; unklar auch Art. 11 VerfEst; Art. 22 VerfEWeißr, Art. 14 Abs. 2 VerfUkr. Dazu kommen dann Regelungen der Grundrechtsgeltung für den Ausnahmefall, siehe Art. 56 nNVerfRF; Art. 44 VerfKas; Art. 139 Verfest; Art. 63 VerfEWeißr; Ar. 14 Abs. 4 VerfEUkr; keine Vorschrift enthält insoweit die Verfassung Usbekistans.

36 Besonders wichtig im Hinblick auf die Vergangenheit sind hiernatürlich die Justizgrundrechte, die insgesamt über den in westlichen Verf assungen garantierten Standard hinausgehen. So finden sich neben den klassischen Justizgrundrechten zumeist auch eine Rechtsschutzgarantie, ein Anspruch auf Schadensersatz für erlittenes Unrecht und ein Recht auf juristischen Beistand sowie zum Teil ein Recht auf Instanzenzug und auch auf Geschworenengerichte: siehe etwa zur Rechtsschutz- 
sungen nicht oder noch nicht vorhanden sind. Die postsozialistischen Verfassungen betonen zum einen (nicht überraschend) bei einigen Grundrechten, so etwa der Meinungsäußerungsfreiheit und der Vereinigungsfreiheit die negative Freiheit, die Möglichkeit also, von dem Grundrecht keinen Gebrauch zu machen. 37 Darüber hinaus geben die Projekte durchgängig dem Bürger sehr weitgehende Informationsansproche zumindest gegenüber dem Staat, zum Teil aber auch gegenüber gesellschaftlichen Organisationen. 38 Beim Grundrecht der Glaubensfreiheit - das nomalerweise ein Unterfall des Grundrechts der Gewissensfreiheit ist überrascht und erfrischt die Betonung der Tatsache, daB auch der Atheismus schutzbedürftig und verbreitenswert ist. ${ }^{39}$ Bemerkenswert, daB zumeist keine Hemmungen bestehen, ein Grundrecht auf Umweltschutz und ein Grundrecht auf Kulturgenuß (an den nationalen Kulturgütern) festzuschreiben. 40 Im übrigen findet sich meist auch ein Recht auf Teil-

garantie: Art. $46 \mathrm{nVerfRF}$; Art. 45 VerfERF/OS; Art. 36 VerfKas (nicht als subjektives Recht formuliert), siehe aber Art. 40 VerfKas; Art 44 VerfUs; Art. 61 VerfEWeiBr; Art. 58 Abs. 1 VerfEUkr. Schadensersatz: Art. 53 nVerRF; Art. 43 Abs. 3 Satz 2 VerfERF/OS; Art. 25 VerfEst; Art. 61 Abs. 2 VerfEWeißr; Art. 58 Abs. 2 VerfEUkr. Juristischer Beisland: Art. 48 Abs. 1 nVerfRF; Art. 44 VerfERF/OS; Art. 43, 105 VerfKas; Art. 62 Abs. 1 VerfEWeiBr; Art. 59 VerfEUkr, Art. 116 VerfUs. Instanzenzug: Art. 45 Abs. 3 VerfERF/OS; die alte Verfassung enthielt dieses Recht nur bei straf rechtlichen Verurteilungen, Art. 65 Abs. 2 aVerfRF; Art. 24 Abs. 5 VerfEst. Geschworenengericht: Art. 47 Abs. 2 nVerfRF. - Siehe im übrigen die Regelungen zum Opferschutz in Art. 52 VerfRF; als allgemeiner Schadensersatzanspruch für rechtswidrige Schäden enthielt diese Regelung auch Art. 43 Abs. 3 Satz 1 VenfERF/OS.

37 Art. 29 Abs. 3 nVerfRF; Art. 25 Abs. 1 Satz 2 VerfERF/OS; Art. 33 Abs. 2 VerfEWeißr; Art. 10 Satz 2 VerfKas; ohne Betonung dieser negativen Freiheit etwa die Verfassung Estlands. - Auch sonstige negative Freiheiten werden hier betont, besonders etwa die negative Vereinigungsf reiheit: siehe Ar. 30 Abs. 2 nVerfRF; wortgleich Art. 32 Abs. 2 VerfERF/OS; Art. 31 Abs. 2 VerfUkr.

38 Art. 24 Abs. 2 nVerfRF, freilich mit einfachem Gesetzesvorbehalt; ähnlich Art. 22 Abs. 5 VerfERF/OS; siehe auch Art.25 Abs. 2 VerfERF/OS; Art. 44 Abs. 2, 3 VerfEst; Art. 33 Abs. 3 VerfKas, formuliert wiederum nicht als Recht, sondem als Pflicht der Staatsorgane; Art. 30 VerUs; Art. 34 VerfWeißr; Art. 30 VerfEUkr. Daneben ist auch die allgemeine Informationsfreiheit durchgängig garantiert, siehe Art. 29 Abs. 4 nVerfR; Art. 25 Abs. 2 VerfERF/OS, - siehe auch dort in Art. 69 Abs. 2 die Garantie freier wissenschaftlicher Information -; Art. 11 VerfKas; Art. 44 Abs. 1 VerfEst; Art. 29 VerfUs; Art. 28 Abs. 2 VerfEUkr. Interessant ist schließlich, daß wiederum in Abkehr von der Vergangenheit einige Verf assungen ein Verfügungsrecht über jegliche persönliche Information enthalten, siehe etwa Art. 24 Abs. 1 nVerfRF; ähnlich Art. 22 Abs. 3 VerfERF/OS; Art. 27 VerfEUkr; besonders ausführlich Art. 41, 42 VerfEst.

39 Art. 28 nVerfRF; wortgleich Art. 26 VerfERF/OS; ohne Betonung des Atheismus Art. 40 VerfEst; Art. 31 LVerfEWeißr; Art. 28 VerfEUkr; Art. 12 VerfKas; Art. 31 VerfUs.

40 Art. 42 VerfRF, mit einem Umweltinformationsrecht und Schadensersatzanspruch; dem entspricht die rechtliche Verantwortlichkeit für die Unterdrückung gesundheitsrelevanter Informationen für Amtspersonen in Art. 41 Abs. 3 nVerfRF; identisch die Ar. 38, 37 Abs. 3 VerfERF/OS; siehe weiter Art. 26 VerfKas (Umweltschutz); Art. 45 Abs. 2 VerfEWeißr, als Teil des Rechts auf Gesundheit; Art. 47 VerfEUkr. Kultur: Art. 44 Abs. 2 nVerfRF; identisch mit Art. 42 Abs. 2 VerfERF/OS; siehe auch die objektiv rechtliche Seite in Art. 70 VerfERF/OS; Art. 51 VerfEWeißr; Art. 49 VerfEUkr; mit korrespondierender Kulturpflicht des Staates vor allem Art. 93, 94 - Rückbringungspflicht für ausländische Kulturschätze. 
nahme an den staatlichen Angelegenheiten und ihrer Entscheidung - dies ist natürlich beste sozialistische, freilich nie verwirklichte Tradition. 41

Bei den sozialen Grundrechten und bei der Freiheit von Eigentum und Untemehmertum zeigen alle Verfassungsentwürfe. und Verfassungen postsozialistisch-kapitalistische Schachbrett-Flagge. Sämtliche Verfassungen enthalten einen ausgedehnten Katalog sozialer Leistungsrechte, so das Recht auf Arbeit, auf Urlaub, auf medizinische Versorgung, auf gesunde Arbeitsbedingungen, auf Mindestlohn usw. Sehr problematisch und in ihrer Absage an die Vergangenheit unterschiedlich dezidiert ist die Regelung der wirtschaftlichen Grundrechte. Ublicherweise findet sich ein Grundrecht des Eigentums - freilich zum Teil in eigenartiger Formulierung -, ein Grundrecht des freien Untemehmertums und auch zum Teil die Eigentumsfähigkeit von Grund und Boden:42 Diese Positionen werden aber in unterschiedlicher Intensität mit sozialen Pflichten und Beschränkmgen von Monopolen und dem Gebot des lauteren Wettbewerbs beschwert. 43 Letztlich machen alle Verfassungen klar, daß man die freie bzw. soziale Marktwirtschaft gutheißt, aber dabei natürlich davon ausgeht, daß es sich um eine anständige Marktwirtschaft handeln wird: Diese verfassungsgebende Blauäugigkeit steht in eigentümlichen Gegensatz zu dem beinharten ManchesterKapitalismus, der sich in der gesellschaftlichen Wirklichkeit dieser Staaten abspielt, soweit es dort entweder zu Kapitalismus oder zumindest zu staatlich nicht kontrollierten, wenn auch nicht unbedingt nicht-staatlichen Monopolen gekommen ist: (Das zweite ist so selten nicht!) Ebenso blauäugig ist natürlich die simultane Normierung von Marktwirtschaften und solchen sozialen Grundrechten - wie etwa dem Recht auf Arbeit -,44 die ein Eigentum des Staates an den Produktionsmitteln voraussetzen. Erklärt wird dies immer wieder damit, daß ein Verfassungsprojekt ohne ein Recht auf Arbeit nicht konsensfähig sei.

41 Art. 32 Abs. 1 nVerfRF; wortgleich Art. 28 VerfERF/OS; siehe weiter Art. 37 VerfEWeißr; Art. 34 VerfEUkr, Art. 14 VerfKas; Art. 32 VerfUs.

42 Eigentum: Art 35 nVerfRF (systernatisch nach der untemehmerischen Freiheit); ähnlich Ar. 35 VerfERF/OS, siehe dazu jedoch auch die objektivrechtliche Seite im Kapitel "Die bürgerliche Gesellschaft", Art. 57 VerfERF/OS; siehe weiter Art. 18 VerfKas (Recht, Eigentümer zu sein) sowie Art. 45, 47 VerfKas (das Eigentum); Art. 36 VerfUs (inklusive des Bankgeheimnisses); Art. 32 VerEst; Art. 44 VerfEWeißr; Art. 36, 66 VerfEUkr. Unternehmertwm: Art. 48 VerfKas (die Freiheit des Untemehmerums); Art. 34 Abs. 1 nVerfRF; Art. 61 VerfERF/OS; Art. 31 VerfEst; Ar. 38 VerfEUkr, siehe ebenda auch die Art. 74 ff.; Ar. 53 VerfUs, kein subjektives Recht. Grund und Boden: Art. 36 Abs. 1 nVerfRF; windelweich Art. 58 VerfERF/OS; sehr unklar auch Art. 55 VerfUs; Art. 67, 68 VerfEUkr, mit erheblichen Restriktionen bei der Eigentumsfähigkeit von Naturschätzen.

43 Art. 34 Abs. 2 nVerfRF (Untemehmertum); Art. 36 Abs. 2, 3 nVerfRF (Grenzen der Nutzung von Grund und Boden); ähnlich Art. 61, 58 VerfERF/OS; Art. 44 Abs. 4 VerfEWeißr, Ar. 36 Abs. 3 sowie Art. 74 ff. VerEUkr; Art. 18 Abs. 3 sowie Art. 48 Abs. 2 VerfKas; Art. 54 Satz 2 VerfUs.

44 Sehr sybillinisch Art. 37 Abs. 1 Satz 3 nVerfRF: Freiheit der Arbeit / Recht auf bestimmte Arbeitsbedingungen; anders - Recht auf frei gewählte Arbeit - Art. 36 Abs. 1 VerfERF/OS; Art. 41 VerfEWeißr; Art. 19 VerfKas (Abs. 2 Freiheit des Arbeitsvertrages; Abs. 3 staatliche Vorgaben für Arbeitsschutz); Art. 37 VerfUs: Recht auf Arbeit und Berufsfreiheit; Art. 39 VerfEUkr. 
In die gleiche Richtung geht die Normiernng von Grundpflichten, die immer für sozialistische Verfassungen besonders typisch war. Kleinster gemeinsamer Nenner ist die allgemeine Wehrpflicht und die Pflicht, Steuem zu zahlen, die sich in allen Verfassungsentwürfen findet; daneben werden angeboten etwa eine Pflicht, die Kulturgüter zu wahren, eine Pflicht, die Natur zu schützen, eine allgemeine Gehorsamspflicht gegenüber den Gesetzen und der Verfassung und gar eine Pflicht, den Mitbürger zu achten.45 Bei so viel Anknüpfung an die sozialistische Vergangenheit benhigt den Leser dann doch, daB andererseits klare Grenzlinien gezogen werden: das Verbot der Ausbürgerung, das Verbot der Aussiedlung und Aberkennung der Staatsangehörigkeit sowie das Recht der Freizügigkeit und die Ausreisefreiheit gehören ebenfalls zum allgemeinen Grundbestand. 46

\section{Präsident und Parlament}

Die Regelung der Beziehungen zwischen Präsident und Parlament ist der Bereich, indem die unterschiedlichen Verfassungsprojekte die größten Unterschiede aufweisen. Dies hat mehrere Gründe. Zum einen geht es hier (anders als bei den Grundrechten) um die Verteilung realer Macht im politischen System; deswegen ist dieser Bereich besonders umstritten.

45 Steuern: Art. 57 nVerfRF; Art. 53 VerfERF/OS; Art. 30 VerfKas; Art. 62 VerfEUkr; Art. 51 VerfUs. Achtung der Gesetze: Dies fehlt in der neuen Verfassung der Russischen Föderation, siehe aber Art. 49 VerfERF/OS; Art. 52 VerfEWeißr; Art. 20,60 VerfEUkr; Art. 29 VerfKas, Satz 2 normiert die Pflicht der Achtung der Staatssymbole! Art. 48 VerfUs; Art. 19 VerfEst, siehe auch Art. 55: die gleiche Verpflichung für Ausländer. Wehrpflicht: Sehr häufig wird vor der Wehrpflicht noch die "Schuld der Verteidigung des Vaterlandes" nomiert: Art. 55 Abs. 1, 2 VerfERF/OS; Art. 31 VerfKas; Art. 57 VerfEWeißr; Art. 52 VerfUs; Art. 59 Abs. 2, 3 nVerfRF, Wehrpflicht und Wehrdienstverweigerung; unklar Art. 54 VerfEst, Treue zur Verfassung; klar wieder Art. 61 VerfEUkr. Umweltschutzpflicht: Art. 58 nVerfRF; Art. 51 VerfERF/OS; Art. 53 VerfEst; Art. 55 VerfEWeißr; Art. 63 VerfEUkr, Art. 50 VerfUs. Denkmalschutzpflicht: Art. 44 Abs. 3 nVerfRF; Art. 52 VerfERF/OS; Art. 54 VerfEWeißr; Art. 63 VerfEUkr; Art. 49 VerfUs. Achtung der Mitbürger und sonstiges: Art. 19 Abs. 2 VerfEst; Art. 53 VerfEWeißr; Art. 48 VerfUs; Art. 29 VerfKas; siehe auch Art. 54 VerfERF/OS, Geschworenenpflicht; Art. 28 VerfKas, Junktim von Rechten und Pflichten.

46 Freizügigkeit: Art. 27 Abs. 1 nVerfRF; wortgleich Art. 24 Abs. 1 VerfERF/OS; Art. 26. VerfEUkr; Art. 13 VerfKas; Art. 34 VerfEst; Art. 30 VerfEWeißr; Art. 28 VerfUs; zumeist sind die Freizügigkeitsrechte als Menschen- und nicht als Bürgerrechte formuliert. Ausbürgerung: Art. 6 Abs. 3 nVerfRF, siehe auch Art. 61 nVerfRF, Verbor der Ausweisung russischer Bürger; ähnlich Art. 16 Abs. 2 u. 3 VerfERF/OS; Art. 15 VerfEUkr, mit einem Verbor der doppelten Staatsbürgerschaft, Abs. 1, 2; Art. 4 Abs. 2 VerfKas; sehr eigenartig Art. 8 Abs. 3, 4 VerfEst: Verbot der Entziehung der angeborenen Staatsbürgerschaft / Verbot der Entziehung der Staatsbürgerschaft wegen der Überzeugung des Betreffenden; Art. 36 VerfEst: Ausweisungsverbote / Siedlungsfreiheit in Estland für Esten. Ausreisefreiheit: Art. 27 Abs. 2 nVerfRF (schrankenlos); wortgleich Art. 24 Abs. 2 VerfERF/OS; Art. 35 VerfEst; Art. 13 VerfKas; Art. 28 VerfUs. - Siehe weiter auch Art. 4 VerfKas: Recht auf Änderung der Staatsbürgerschaft; Art. 62 nVerfRF: Möglichkeit der Doppelstaatsbürgerschaft; fast identisch Art. 18 VerfERF/OS. 
Dazu kommt, daß die Verfassungstradition in allen untersuchten Ländern durch eine starke Exekutive (sei es in Form der Exekutive, sei es in Form der kommunistischen Partei) gekennzeichnet ist und da $B$ man sich deshalb gerade für die schwierigen Zeiten einen starken Mann an der Spitze des Staates wünscht. Konkret hat zumeist der ehemalige Vorsitzende des Obersten Sowjets auf diese oder jene Weise das Präsidentenamt übernommen. Andererseits sehen sich die Parlamente in der Tradition der Räte und gehen deswegen von verschwommenen Vorstellungen von Allkompetenz aus. Schließlich leistet noch das russische Beispiel, an dem man sich allgemein orientiert, seinen Beitrag zu der allgemeinen Verwirmung. Bezeichnend ist, daB wir nur in Estland ansatzweise eine parlamentarische Demokratie finden; alle anderen Systeme sind Präsidialdemokratien. Im einzelnen kreisen die Streitigkeiten der Machtverteilung zwischen Präsident und Parlament um folgende Fragen: Soll der Präsident Staatsoberhaupt oder soll er Spitze der Exekutive sein?47 Soll der Präsident die alleinige Kompetenz haben, die Regierung zu bilden und zu entlassen, oder aber soll das Parlament bei der Regierungsbildung durch ein Zustimmungsrecht mitwirken dürfen48 und soll das Parlament gegenüber der Regierung - der gesamten Regierung oder auch einzelnen Ministem - ein Mißtrauensvotum haben?49 Soll der Präsident die Möglich-

47 Art. 80 nVerfRF "Staatsoberhaupt", aber im materiellen Sinne; siehe demgegenüber Art. 93, 85 VerfERF/OS: Präsident als Staatsoberhaupt / Parlament als einziges gesetzgebendes und vertretendes Organ. Art. 75 VerfKas: Staatsoberhaupt und Spitze der Exekutive; siehe dagegen Ar. 62 VerfKas: Parlament als höchstes vertretendes und einziges gesetzgebendes Organ; ebenso Ar. 89 VerfUs. Interessant hier Ar. 97 VerfUs: Nach der Amtszeit wird der Präsident autornatisch lebenslang Verfassungsrichter. Art. 77 VerfEst: Staatsoberhaupt; Art. 175 VerfEUkr, Art. 95 VerfEWeißr: Beide Staatsoberhaupt und Spitze der Exekutive.

48 Art. 83a nVerfRF: Bestimmung des Ministerpräsidenten mit Zustimmung des Parlaments; Art. 83w gibt das Recht der Regierungsauflösung dem Präsidenten ohne Mitwirkung des Parlaments; darüber hinaus schlägt der Präsident noch den Vorsitzenden der Staatsbank und die Obersten Richter sowie den Generalstaatsanwalt vor, Art. 83g, 83e nVerfRF. - Siehe demgegenüber den VerfERF/OS Ar. 87 Abs. 1w: Kompetenz der grundlegenden innen- und außenpolitischen Entscheidungen; Art. 87 Abs.11: weitgehende Bestimmungsrechte bei Regierungsbildung gegenüber Art. 94 Abs. lb für Präsidenten; Art. 94 Abs. 1d gibt das Vorschlagsrecht der Obersten Richter, des Generalbundesanwalts und des Vorsitzenden der Staatsbank; ähnlich Art. 64 Nr. 10, 28 Abs. 1, 3 VerfKas; Art. 78 Nr. 16 VerfUs: die gesamte Regienung; siehe auch die Regierungsorganisationskompetenz in Nr. 19, ebenda; Art. 140 VerfEUkr, Art. 84 Abs. 1 Nr. 7 VerfEWeißr, mit sämtliche Hauptministerien umf assenden Zustimmungsrechten.

49 Art. 117 Abs. 3 nVerfRF, freilich entscheidet dann der Präsident über den Rücktritt der Regienung; darüber hinaus kann das Parlament bei wiederholten Mißtrauensvoten vom Präsidenten aufgelöst werden, Art. 117 Abs. 3 Satz 4 nVerfRF; ähnlich schwach Art. 99 Abs. 4 VerfERF/OS; ganz anders Art. 88 VerfKas: Verantwort lichkeit der Minister für den Vollzug der Gesetze / Möglichkeit des Parlaments, beim Präsidenten den Regierungsrücktritt zu fordem. Ein Mißtrauensvotum fehlt gänzlich in Usbekistan, wo freilich der Präsident auch Vorsitzender der Regierung ist; siehe aber demgegenüber das sich auf sämtliche Regierungsmitglieder und die Regierung beziehende Mißtrauensvotum in Art. 65 Nr. 13 VerfEst. 
keit haben, das Parlament aufzulösen und Neuwahlen auszuschreiben? ${ }^{50}$ Soll der Präsident die Möglichkeit haben, ein Referendum zu initiieren, was seine Positionen gegenüber dem Parlament wiederum stärken würde?51 Steht dem Präsidenten eine eigene Dekretmacht zu?52 Kann im Falle der Gesetzes- bzw. Verfassungswidrigkeit eines Dekrets das Parlament dieses aufheben; soll das Gesetz in der Normenhierarchie höher stehen als das präsidiale Dekret?53 Hat der Präsident ein suspensives, ein absolutes oder gar kein Veto gegenüber Gesetzen?54 Wie stark ist die Regienung gegenüber dem Präsidenten, etwa durch die Möglichkeit eigener Gesetzesinitiative etc.?55 Und schließlich die beiden Kardinalfragen:

50 Ar. 84b, 109 nVerfRF, diese Möglichkeit besteht vor allem im Falle des Ar. 111 Abs. 3 bei mißlungener Regierungsbildung; keine Auflösungsmöglichkeit hat der Präsident nach dem VerfERF/OS (und bekanntlich auch nicht nach der alten Verfassung, Ar. 121/6 aVerfRF); ebensowenig die Verfassung Kasachstans, die aber auch kein impeachment kennt; siehe weiter die Möglichkeiten des Art. 95 VerfUs; Art. 181, 187 VerfEUkr: nur bei fehlgeschlagenem Amtsenthebungsverf ahren gegen den Präsidenten; Art. 82 Abs. 2 VerfEWeißr normiert ein Selbstauflösungsrecht des Parlarnents.

51 Ar. 84w nVerfRF; Ar. 91 Abs. 5b VerfERF/OS: bei Unterstïtzung durch 1/3 der Abgeordneten; Art. 64 Nr. 7 VerfKas gibt die Entscheidung dem Parlament, gem. Art. 78 Abs. 1 Nr. 7 entscheidet freilich der Präsident nach Konsultation mit dem Parlament über ein Referendurm; siehe weiter Art. 166 VerfEUkr.

52 Ar. 90 nVerfRF: eigenständige Dekretmacht; ebenso Ar. 94 Abs. 2 VertERF/OS; unklar Ar. 79 VerfKas: auf der Grundlage und in Ausfühnung der Verfassung und der Gesetze; identisch Ar. 94 VerfUs. Nur eine Dekretmacht für Notfalle hat der estnische Präsident, Art. 73 Nr. 7, 109, 110 VerfEst; sehr weitgehend Ar. 182 VerfEUkr, der nur eine Verfassungsbindung der Dekretmacht vorsieht; Ar. 101 VerfEWeiBr: in den Grenzen seiner Kompetenz.

53 Ar. 90 Abs. 3 nVerfRF: Gesetz bricht Ukaz; chenso Ar. 94 Abs. 2 Satz 2 VerfERF/OS; unklar Ar. 79 VerfKas; ebenso Ar. 94 VerfUs. Interessant Ar. 135 VerfEUkr, der für bestimmte Materien der Nationalversammlung die ausschließliche Gesetzgebungskompetenz gibt: Es bleibt unklar, ob diese Ausschließlickeit im Verhältnis zu den beiden Kammem oder zum Präsidenten und seiner Dekretmacht gemeint ist; siehe auch Art. 135 Abs. 3 "Bestätigung sonstiger Gesetze"; siehe im übrigen Art. 138 Abs. 1 Nr. 14 VerfEUkr, parlamentarisches Veto gegen die Dekrete des Präsidenten.

54 Art. 107 Abs. 3 nVerfRF: nur suspensives Veto, freilich kann dies mit 2/3 Mehrheit der Mitglieder überstimmt werden; genauso Art. 90 Abs. 3 VerfERF/OS; Art. 78 Abs. 1 Nr. 2 VerfKas; Art. 93 Abs. $1 \mathrm{Nr}$. 40 VerfUs; kein Vetorecht normier Ar. 73 Nr. 6 VerfEst; ein Vetorecht siehe aber in Art. $178 \mathrm{Nr} .8$ VerfEUkr; Ar. $100 \mathrm{Nr}$. 17 VerfEWeißr, siehe auch ebenda das absolute Veto bei Gesetzen, die im Budget nicht vorgesehene Ausgaben verursachen, Nr. 18. Siehe weiter die Möglichkeit, gesetzeswidrige Entscheidungen von "Repräsentativorganen" zu suspendieren, Ar. 100 Nr. 20: rein theoretisch wäre hier auch das Parlarnent mit Einzelf allentscheidung gemeint.

55 Ar. 104 Abs. 1 nVerfRF: Gesetzesinitiative; siehe darüber hinaus Art. 104 Abs. 3, Stellungnahme bei ausgabewirksamen Gesetzen; siehe demgegenüber Ar. 85 Abs. 2, Möglichkeit des Präsidenten, gesetz- oder verfassungswidrige Akte der Organe der Exekutive zu suspendieren; siehe auch Ar. 110 Abs. 1 nVerfRF: die Regierung "verwirklicht" die Exekutivgewalt, mit einer weiteren Schwächung der Regierung in Ar. 112, 113 nVerfRF. Keine Gesetzesinitiative hat die Regierung gem. Ar. 90 Abs. 1 VerfERF/OS; eine weitere Schwächung siehe in Ar. 98 Abs. 4 Satz 2 VerfERF/OS: Abänderung der Regierungsakte durch Präsidenten. Art. 71 VerfKas gibt die Gesetzesinitiative der Regienung; diese ist jedoch gem. Art. 75 faktisch dem Präsidenten unterstellt, 
Soll der Präsident durch das Volk, das Parlament oder eine spezielle Versammlung gewählt werden?56 Unter welchen Voraussetzungen kann er seines Amtes enthoben werden?57

Die Antworten der Verfassungen und Verfassungsentwürfe auf diese Fragen variieren und verändern sich häufig: Während etwa bei den parlamentarischen Verfassungsentwürfen erwartungsgemäß die Vollmachten des Präsidenten eher begrenzt sind, gibt der neue Verfassungsentwurf Jelzins dem Präsidenten und damit ihm selbst nahezu unbegrenzte Machtkompetenz gegenüber dem Parlament.

Daneben fallen noch einige Besonderheiten auf, die zeigen, daB die Funktionsmodi einer präsidialen oder nicht präsidialen - Demokratie wohl teilweise nicht so leicht zu durchschauen sind. In allen Verfassungsentwürfen fällt auf, daB die Parlamente eine eigentümliche Kombination von stark und schwach sind. Sie sind schwach in dem Sinne, daß sie nur begrenzt über die klassischen Instrumente westlicher Parlamente wie etwa Mißtrauensvoten, Untersuchungsausschüsse und eigenstăndige Amtsenthebungsverfahren verfügen. 58

siehe auch Art. 78 Abs. 1 Nr. 3 VerfKas. Art. 82, dann aber 89 VerfUs: Präsident ist gleichzeitig Vorsitzender des Kabinetts; klar auch die Unterordnung der Regierung gegenüber dem Präsidenten in Art. 191 VerfEUkr, ebenso Art. 193 Abs. 3; keine Gesetzesinitiative für die Regierung sieht der weißrussische Verfassungsentwurf vor, Art. 90, wohl aber für die Obersten Gerichte - dies ist üblich und zumeist auch in den anderen Verfassungsentwürfen und Verfassungen $z$ finden - und aber auch für die Staatsbank - dies ist unüblich.

56 Art. 81 nVerfRF: Volk / 5 Jahre; ebenso Art. 76 VerfKas; Rat. 90 Abs. 2 VerfUs; Art. 175 Abs. 3 VerfEUkr; Art. 97 VerfEWeißr. Anders Art. 93 Abs. 2, 4 VerfERF/OS: Volk / 4 Jahre; noch anders die stark parlamentarische Verfassung Estlands: Art. 79: Parlament bzw. - soweit dieses sich nicht einigen kann - Kollegium der Wahtminner, Art. 79 Abs. 4, auf 5 Jahre, Art. 80 VerfEst.

57 Art. 92 Abs. 3, 93 nVerfRF; die Voraussetzungen sind ein schweres Verbrechen oder Staatsverbrechen, eine Anklage der Duma mit 2/3 der Mitgliedermehmeit bei einer Initiative von mindestens 1/3 der Mitglieder; efforderlich ist weiter eine entsprechende Stellungnahme des Obersten Gerichts zum Gegenstand der Anschuldigung und des Verfassungsgerichts zur Einhaltung des vorgeschriebenen Verfahrens. Der Föderationsrat muß dann innerhalb von 3 Monaten wiederum mit 2/3 Mitgliedermehneit entscheiden; das Gesetz erwähnt weiter die Notwendigkeit einer Stellungnahme einer speziellen Kommission. Der Artikel ist gegenüber den beiden vother vorgestellten Verfassungsversionen vollständig verändert und macht die Amtsenthebung gegenüber dem Art. 83 des Aprilentwurfs und Art. 88 des Julientwurfs so gut wie unmöglich, da besonders die Mehrheitserfordemisse beim Antrag und bei der Entscheidung verschärft worden sind. Ar. 96 VerfERF/OS eröffnete das impeachment auch bei groben Verfassungsverletzungen, entsprach im übrigen der April- und Juliversion der Jelzin-Verfassung. Dies wiederum entsprach größtenteils der alten Regelung des Art. 121/10 aVerfRF. - Keine Möglichkeit eines impeachments enthalten die Verfassungen Kasachstans und Usbekistans, abgesehen von der Amtsunfähigkeit aus gesundheitlichen Gründen, siehe etwa Art. 96 VerfUs. Siehe weiter die impeachment-Regelungen in Art. $140 \mathrm{Nr} .6,183,188$ VerfEUkr; hier ist jedoch auch eine Abwahl per Referendum vorgesehen, Art. 183, 186 VerfEUkr. Siehe weiter 104 VerfEWeißr: $2 / 3$ Mehrheit. Ein echtes impeachment fehlt auch in der estnischen Verfassung, siehe Art. 83 VerfEst.

58 Siehe etwa Art. 101 Abs. 3 nVerfRF: "bildet Kommissionen"; eine gewisse destruktive Potenz enthält allerdings Art. 114 Abs. 2 nVerfRF, wonach die Tätigkeit der Regierung nicht etwa durch 
Sie sind andererseits stark in dem Sinne, daB ihnen nicht nur die gesetzgebende und die kontrollierende Funktion gegeben wird, sondern normalerweise auch ausdrücklich die Möglichkeit, nicht nur Gesetze, sondern auch Beschlusse zu erlassen, neben abstrakt generellen auch konkret individuelle Entscheidungen mit Außenwirkung zu fällen.59 Bei überlappenden Kompetenzen mit der Exekutive können die Parlament also, im Sinne der alten Allkompetenz, quasi exekutivische Entscheidungen fällen, da die Grenzlinien der Gewaltenteilung nicht auch in der Rechtsform gezogen werden.

Ein Vermächtnis der Vergangenheit ist auch die Eigentümlichkeit, daß sowohl die Stellung des Präsidenten wie auch des Parlaments in detaillierten Kompetenzkatalogen niedergelegt ist, wobei dann freilich diese Kataloge durch eine "und sonstige Kompetenzen - Klausel" am Schluß ihren enumerativen Sinn wieder verlieren. 60 Es fältt weiterhin auf, daß das Verfassungsrecht Schwierigkeiten hat, den politischen Parteien und gesellschaftlichen Organisationen einen adäquaten Platz zuzuweisen und beide sinnvoll und trennscharf voneinander abzugrenzen.61 Im Bereich des Parlamentsrechts taucht die politische Partei überhaupt nicht auf; die allgemeinen Regelungen werfen politische Parteien und gesellschaftliche Organisationen in einen Topf und verkennen so die besondere Rolle der politischen Parteien bei der Vorstrukturierung des politischen Willens. Zum Teil ging und geht das Durcheinander sogar soweit, daß man bestimmten gesellschaftlichen Organisationen wie etwa

deren Geschäftsondnung, sondem durch ein Verfassungsgesetz geregelt wird. Siehe weiter Ar. 87 Abs. 1b, 89b VerfERF/OS; Ar. 87 VerfUs; Ar. 71, 74 VerfEst; siehe andererseits auch die ausdrückliche Regelung der Kontrollfunktion des Parlaments bezüglich Exekutive und Judikative in Ar. 136 Nr. 3 VerfEUkr sowie ebenda auch die Rechte des Arr. 140, 153; sehr kurz wiederum Art. 86 Abs. 1, 2 VerfEWeißr.

59 Ar. 102 Abs. 2, 103 Abs. 3 nVerfRF; Ar. 87 Abs. 2 VerfERF/OS; Ar. 64 Abs. 1 Nr. 2 VerfKas; Art. 83 VerfUs; Art. 65 Nr. 1 VerfEst; Ar. 135, 138 Abs.1 Nr.19 VerfEUkr; Ar. 84 Abs. 1 Nr. 3 VerfEWeißr.

60 Die neue russische Verfassung ist bezüglich des Präsidenten in den Ar. 83, 84, 85, 86 u. 89 abschließend enumerativ; das gleiche gilt für das Parlament. Siehe demgegenüber Ar. $87 \mathrm{Abs}$. 1t, Ar. 94 Abs. 1s VerfERF/OS; Ar. 64 Nr. 21, 78 Nr. 17 VerfKas (siehe auch das gleiche Prinzip beim Vorsitzenden des Obersten Sowjets, Art. 65 Nr. 6 VerfKas). Siehe weiter Ar. 78 Nr. 24 VerfUs: Auffangkompetenz nur für dasParlament; noch klarer die allgemeine Auffangkompetenz in Ar. $65 \mathrm{Nr} 16$ VerfEst. Ar. 178 VerfEUkr gibe nur dem Präsidenten eine Auffangkompetenz; zwei Auff angkompetenzen regelt wiederum Art. 84 Abs. 3, Ar. $100 \mathrm{Nr}$. 24 VerfEWeißr.

61 Siehe Ar. 13 Abs. 3, 4, 30 nVerfRF; gem. Ar. 13 Abs. 5 sind zwar bestimmte Vereinigungen verboten; das Verfassungsgericht hat jedoch die Kompetenz für Verbote von Parteien und gesellschaftlichen Organisationen, die ihm nach Ar. 165/1 Nr. 1, letzte Variante aVerfRF zustand, verloren, da sie in der neuen Verfassung nicht wieder auftauchen und im Verfassungsgerichtsgesetz nie einf achgesetzlich geregelt war. Art. 62,63 VerfERF/OS; Ar. 63 sprach etwa von Parteien und anderen politischen gesellschafulichen Organisationen. Siehe weiter Ar. 53 ff. VerfKas: Hier gibt immerhin Art. 56 den Parteien eine besondere Funktion; diffus wiedenm Ar. 34 VerfUs; Art. 48 VerfEst; Art. 31, 95 ff. VerfEUkr. 
den Gewerkschaften ${ }^{62}$ parteienähnliche Funktionen, etwa die Aufstellung von Kandidaten für die Wahlen, zubilligen wollte.

\section{Das Gerichtssystem}

Bei den bisher beschriebenen Regelungsmaterien sahen sich die postsozialistischen Verfassungsschreiber regelmäßig mit neuen Aufgaben konfrontiert: Abgesehen von der Regelung der Präsidentschaft in der UdSSR hatte man sich weder über Staatsstrukturbestimmungen einer neuen Zeit noch über ein modernes Grundrechtskapitel oder über die Beziehungen zwischen einem echten Präsidenten und einem echten Parlament besondere Gedanken gemacht. Dies gilt nicht für die Konzeption einer rechtsstaatlichen Anfordenmgen genügenden Gerichtsbarkeit. Nur kurze Zeit nach dem Machtantritt Gorbacevs und der Einleitung der Reformpolitik begann die konzeptionelle Arbeit an der Reform der sowjetischen Gerichtsbarkeit, deren Zustand allgemein als völlig unbefriedigend empfunden wurde.63 Auf diesen Fundus und konkret auch auf die Konzeption der Gerichtsreform, wie sie schon in der UdSSR ausgearbeitet wurde, können bzw. konnten die Verfassungskommissionen der einzelnen Staaten zurückgreifen, und entsprechend glatt, wenn auch von bestimmten Besonderheiten geprägt, sind die Regelungen der dritten Gewalt. Betont wird die Unabhängigkeit der Gerichte sowohl in institutioneller wie in persönlicher, vor allem materieller Hinsicht - ohne daß man es freilich schaffen wüde, den Richter auf Lebenszeit zur Regel zu machen: In einigen Texten finden wir eine 10jährige Amtsperiode.64 Sämtliche Verfassungsentwürfe enthalten die allgemein akzeptierten rechtsstaatlichen Grundsätze des gesetzlichen Richters, des Rückwirkungsverbots, des rechtlichen Gehörs usw. Durchgängig finden wir auch - allerdings normalerweise im Grmdrechtskapitel - eine dem Artikel 19 IV GG vergleichbare Rechtsschutzgarantie, die darüber hinaus noch durch ein Grundrecht auf juristische Beratung und juristischen Beistand abgesichert ist.65 In gleicher Weise hat die konkrete Normenkontrolle in Form einer Richtervorlage die Verfassungsgeber der postsozialistischen Länder überzeugt: Fast alle Verfassungen weisen in irgendeiner Form eine

62 Dies taucht in den veröffentlichten Verfassungsentwürfen nicht auf; ich selbst war jedoch bei einer Beratungssitzung der Verfassungskommission zugegen, wo den Gewerkschaften in alter Tradition das Recht der Kandidatenaufstellung für die Wahl gegeben wurde; diese Vorschrift wurde erst einige Zeit später wieder gestrichen.

63 Siehe dazu meine Darstellung in: "Rechtsstaat UdSSR", Jahßuch für Ostrecht, XXXV1 (1990), S. 9 (23 ff.).

64 Sehr fortschrittlich die Ar. 120 (Unabsetzbarkeit), 121 (Immunität) sowie 124 (ausreichende finanzielle Ausstattung) nVerfRF; ähnlich Art. 106 VerfERF/OS; Ar. 100 VerfKas: 10 Jahre; siehe aber Art 103 / Immunität, 104 / materielle Ausstattung; Art. 107 VerfUs: 5 Jahre; Art. 112: Unabhängigkeit und Immunitä; Art. 147 VerfEst: Lebenszeit; eine begrenzte Immunität findet sich in Ar. 153 VerfEst. Art. 207 bis 209 VerfEUkr: auf Lebenszeit nach einer Probezeit von 5 Jahren; Ar. 114 VerfEWeißr: auf Lebenszeit.

65 Siehe die Nachweise in Anmerkung 36. 
Vorlagemöglichkeit an das Verfassungsgericht auf, wenn ein Gericht ein Gesetz oder eine andere Norm bei einer Entscheidung für verfassungswidrig hält.66 Ähnlich einheitlich ist leider die Ablehnung eines umfassenden direkten Zugangs des Bürgers zum Verfassungsgericht im Falle einer möglichen Grundrechtsverletzung: Zum Teil gab oder gibt es freilich nicht unoriginelle Altemativen, wie etwa die Überprüfung einer Rechtsanwendungspraxis oder aber auch einen Bürgerrechtsbeauftragten (im Entwurf der Verfassungskommission des Obersten Sow jets der Russischen Föderation). 67

Im übrigen ist in den meisten Fällen, sowjetischer Tradition folgend, die Tätigkeit (nicht nur) der Verfassungsgerichte mit einigen Fragezeichen zu versehen. $\mathrm{Zu}$ den bekannten Problemen kommt noch eine spezifische sowjetische Vorstellung der wesensmäßigen Unterschiedlichkeit von Gesetzesauslegung und Gesetzesanwendung: Die Gesetzesauslegung, vor allem aber auch die Verfassungsauslegung war (und ist teilweise noch) eine Kompetenz des Obersten Sowjets bzw. des Parlaments;68 dies läßt es als möglich erscheinen, daß die Verfassungsgerichte aus funktionell-rechtlichen Grinden sich schwertun werden, jenes $\mathrm{MaB}$ an richterlicher Rechtsschöpfung zu verwirklichen, ohne da $B$ nach bundesrepublikanischem Rechtsdenken weder ein effizientes Gerichtssystem noch eine flexible Anpassung der Rechtsordnung an den sozialen Wandel vorstellbar ist.

66 Art. 125 Abs. 4 nVerfRF; Art. 106 Abs. 4 VerfERF/OS, interessanterweise systematisch in der Regelung der richterlichen Unabhängigkeit; Art. 101 Abs. 2 VerfKas; Art. 217 VerfEUkr, Art. 115 Abs. 2 VerfEWeißr. Unklar ist die Regelung in der estnischen Verfassung: Art. 152 regelt ein Anwendungsverbot für verfassungswidrige Gesetze für alle Gerichte und eine Verwerf ungsmöglichkeit für das Oberste Gericht (Supreme-Court-Modell), ohne jedoch eine Vorlage zu erwähnen; einen Weg zum Obersten Gericht hat nur der "Kanzler der Justiz", Art. 139 ff., 142 VerfEst. Ohne Regelung ist insoweit die Verfassung Usbekistans.

67 Art. 125 Abs. 4 nVerfRF; im Gegensatz zu der alten Regelung des Art. 165/1 Abs. 1 vorletzte Variante aVerfRF wird aber nicht mehr die Verfassungswidrigkeit der Rechtsanwendung - nach der alten Regelung eine Entscheidung nur insoweit sie einer allgemeinen Praxis entsprach geprüft, sondem die Verfassungsmäßigkeit des entscheidungserheblichen Gesetzes. Ähnlich anscheinend Art. 243 Abs. 3 VerfEUkr; anders: (Überprüfung der Rechtsanwendungspraxis) Art. 101 Abs. 2 Satz 2 VerfERF/OS; sehr unklar Art. 130, 131 VerfKas: Es wird eine Klage, aber nicht der mögliche Kläger genannt. Keine Regelung enthält die Verfassung Usbekistans sowie der Verfassungsentwurf Weißrußlands. - Einen Ombudsmann für Menschenrechte sah Art. 48 VerfERF/OS vor, Abs. 3 im übrigen die Möglichkeit eines Minderheitenbeauftragten; im Gegensatz zu früheren Versionen gibe der Entwurf des Sommers 1993 dem Ombudsmann jedoch kein Antragsrecht beim Verfassungsgericht.

68 Siehe etwa Art. 109 Nr. 8 aVerfRF, authentische Auslegung der Gesetze der RF durch den Obersten Sowjet; für den Volksdeputiertenkongreß fehlte für die Verfassung eine ausdrückliche Kornpetenz der authentischen Auslegung; sie dürfte sich jedoch aus Art. 104 Abs. 2, 3 Nr.1 implizit ergeben haben. Siehe jetzt Art. 125 Abs. 5 nVerfRF: Kompetenz der Verfassungsauslegung für das Verfassungsgericht; ähnlich (Verfassung und Gesetz) Art. 109 Abs. 1 Nr. 3 VerfUs; die authentische Auslegung durch das Parlament enthalten noch Art. 64 Abs. 1 Nr. 3 VerfKas; Art. 84 Abs. 1 Nr. 4 VerfEWeißr. 
Eine weitere Besonderheit stellt die Altlast der Prokuratur dar. Wiederum im sowjetischen Recht hatte die Staatsanwaltschaft oder Prokuratur eine Doppelfunktion: Neben der Strafverfolgung oblag ihr die allgemeine Rechtsaufsicht über die Rechtmäßigkeit des staatlichen Handelns; der Bürger konnte diese Kontrollprozedur initiieren und in der Praxis funktionierte, relativ gesehen, die Prokuratur als Kontrollinstitution nicht schlecht und zumindest bedeutend besser als die Gerichtsbarkeit.69 Im Zuge der Reformen wollte man der Prokuratur die allgemeine Rechtsaufsicht nehmen und diese Kontrollfunktion den Gerichten übertragen; die Prokuratur und vor allem viele der älteren Juristen, die an den Reformprojekten mitarbeiten, haben jedoch andere Vorstellungen, so daB in fast allen Texten die Duppelfunktion der Prokuratur noch zu finden ist. Dies bedeutet dann freilich im Endeffekt, $\mathrm{daB}$ die Verwaltung sowohl von der Gerichtsbarkeit (als zwingende Folge des allgemeinen Anspruchs auf Rechtsschutz) wie auch von der Prokuratur kontrolliert wird.

Erwähnenswert ist zu guter Letzt die sich von westlichen Vorstellungen unterscheidende funktionale Differenzienung innerhalb der Gerichtsbarkeit: Keiner der Verfassungsentwürfe sieht eine Verwaltungsgerichtsbarkeit vor; daftr enthalten fast alle Verfassungentwürfe ein Wirtschaftsgericht als Nachfolger des Wirtschafts- oder Arbitragegerichts, das für die Streitigkeiten zwischen den Staatsuntemehmen im Bereich der Planerfüllung zuständig war. 70

\section{Kommunale Selbstverwaltung und Finaveen}

Alle Verfassungen bzw. Verfassungsentwürfe führen die Selbstverwaltung auf örtlicher Ebene ein bzw. wollen sie einführen; allgemein ist auch eine große Ungeschicklichkeit bei der Verwirklichung dieser Absicht. In der Regel wird den örtlichen Einheiten die Selbstbe-

69 Ar. 129 nVerfRF; Art. 129 Abs. 5 überläBt die Bestimmung der Kompetenz dem (Bundes-) Gesetzgeber; Art. 109 VerfERF/OS begrenzt die Aufsicht auf die Gesetzlichkeit (Abs. 3), machte freilich gleichzeitig die Prokuratur zum Vertreter der Anklage (Abs. 2); immerhin war hier die im Augenblick nur einfachgesetzlich abgesicherte Unabhängigkeit der Prokuratur in der Verfassung festgeschrieben, Art. 109 Abs. 4. Ganz herkömmlich Art. 106 ff. VerfKas, mit der Regelung der Unabhängigkeit in Art. 107; Art. 118 ff. VerfUs mit einer sehr eigenartig formulierten Unabhängigkeit in Art. 120; Art. 220 ff. VerfEUkr, freilich ohne eine Garantie der Unabhängigkeit; Art. 136 VerfEWeißr, ohne Absicherung der Unabhängigkeit. Die estnische Verfassung hat einen Ombudsmann, den "Kanzler der Justiz", Art. 139 ff. VerfEst, der freilich Auf sichtsfunktionen hat.

70 Art. 127 nVerfR; freilich erwähnt Art. 118 Abs. 2 nVerfRF die verwaltungsrechtliche Gerichtsbarkeit; da von einem Verwaltungsgericht nirgendwo die Rede ist, dürften wohl wie bisher die ordentlichen Gerichte die gerichtliche Kontrolle der Verwaltung durchführen. Ähnlich Art. 100 Abs. 1 Satz 2, 102, 103 VerfERF/OS; Art. 95 Abs. 4 VerfKas; Art. 107 Abs. 3 VerfUs; Art. 203, 205 VerfEUkr / ordentliche Gerichte und Wirtschaftsgerichte, die estnische Verfassung hat gem. 148 Abs. 1 Nr. 1 auf der untersten Gerichstsstufe auch Verwaltungsgerichte; keine Regelung enthält der weißrussische Verf assungsentwurf. 
stimmung in den örtlichen Angelegenheiten garantiert; die sonstigen Regelungen machen jedoch klar, daß hier, abgesehen einmal von der Kompetenz im (Staats)Wirtschaftswesen, wenige originäre örtliche Kompetenzen begrindet werden.71 Bezeichnend ist, daß die Finanzierung der Kommunen über einen Anteil am Gesamtsteueraufkommen nicht garantiert wird und daß die Verfassungen, durch detaillierte Regelung von Einzelheiten, die kommunale Selbstverwaltung faktisch zu einer Kompetenz der Föderation bzw. des Gesamtstaates machen.

Ähnlich lückenhaft ist die Regelung des Finanzsystems: Festgelegt wird das Erfordemis eines Haushaltsgesetzes und die Finanzienmg des Staates über Steuem, sonstige öffentliche Abgaben und aus seiner wirtschaftlichen Tătigkeit.72 Irgendwelche Grundsätze für die Besteuerung sehen die Texte nicht vor; die im Augenblick existierenden Steuergesetze etwa in der Russischen Föderation sind denn auch beim Entstehen eines markwirtschaftlichen Systems alles andere als hilfreich. In gleicher Weise fehlt für die Russische Föderation jegliche Regelung für die Verteilung des Steveraufkommens zwischen dem Zentrum und den Republiken bzw. Gebieten. Auch hier scheint es schwer zu sein, sich von der Vorstellung eigener wirtschaftlicher Tätigkeit des jeweiligen Trägers hoheitlicher Gewalt zu lösen: $\mathrm{Da} B$ diese Vorstellungen noch fortdauern, macht etwa auch die Ausdifferenzierung der Eigentumsformen nach Eigentumsträgern klar.73

\section{Besonderheiten posecozialistischer Verfassungsgebung}

Wir haben schon gesehen, daB bei aller Orientierung an westlichen Vorbildem, die Verfassungsprojekte inhaltlich gewisse Schwerpunktsetzungen und Besonderheiten aufweisen, die uns fremd sind. Es sind dies zum einen inhaltliche Absagen an die Vergangenheit, wie das Verbot der Ausbürgerung, die man mit Verständnis und Freude liest; andererseits findet man jedoch auch noch jede Menge gegenwärtiger, nur zum Teil erfreulicher Vergangenheit. Erwähnt seien hier noch einmal die Betonung sozialer Grundrechte, sozialer Bindungen von Abwehrrechten, eine gewisse Unbekümmertheit um die praktische Realisierung

71 Siehe Art 130 ff. nVerfF, ohne besondere Eingriffsrechte; siehe dazu aber noch das Verwaltungssystem in den Art. 77, 78. Art. 112 ff. VerfERF/OS, besonders Art. 113; zur alten Rechtslage vgl. Art. 138 ff. aVerfRF und das teilweise noch geltende Gesetz zur örtlichen Selbstverwaltung, siehe dazu das Gesetz mit Einleitung von O. Baller im Jahrbuch für Ostrecht (JOR), XXXII/2 (1992), S. 365 ff.; Art. 90 ff. VerfKas nach Maßgabe der Gesetze; Art. 99 ff. VerfUs; Art. 154 ff. VerfEst, siehe besonders Art. 156 ff.; Art. 120 ff., besonders Art. 123 ff. VerfEWeißr; Art. 224 ff. VerfEUkr.

72 Die neue Verfassung der Russischen Föderation regelt die Finanzen im Föderationskapitel, Art. 75; Art. 115 ff. VerfERF/OS; noch karger war die Regelung in Art. 158 ff. aVerfF; Art. 119 ff. VerfKas; Art. 122 ff. VerfUs; Art. 111 ff. Verfst; Art. 144 VerfEWeißr; Ar. 167 ff. VerfEUkr im Abschnitt über die Legislative.

73 Siehe die Nachweise in Anmerkung 40, 41. 
von verfassungsrechtlich garantierten Rechtspositionen, eine simultane Faszination durch präsidial-autoritäre und räte/direktdemokratische Elemente, wenig Rezeptivität für den zugegebenermaßen spröden Charme der Verwaltungsgerichtsbarkeit sowie eine hilflose Ergebenheit gegenüber der Erblast der Prokuratur. Unterstrichen sei in diesem Zusammenhang weiter die betrübliche und hoffentlich folgenlose Besonderheit, daß man theoretisch Nationalitäts- und Souveränitätsvorstellungen des 19. Jahrhunderts nachhängt und praktisch auch durchaus geneigt zu sein scheint, diese in die Tat umzusetzen, wenn es denn sein muß. In ganz anderem Zusammenhang paßt zu diesen sehr traditionalistischen Vorstellungen das hilflose "Ja, aber" zur Todesstrafe.74

Die Verfassungen und -entwürfe sind sich im übrigen untereinander ziemlich ähnlich; wirkliche Unterschiede ergeben sich vor allem bei der Regelung der Machtverteilung im politischen System. Dies gilt auch und vor allem für die alte und neue Verfassung der Russischen Föderation sowie für den Verfassungsentwurf des Obersten Sow jets: Die Grundrechtskataloge der drei Verfassungen sind mehr oder weniger gleich; der Rechtsschutz, vor allem die Verfassungsgerichtsbarkeit, ist in den Verfassungen der "Verlierer" stärker ausgeformt, ebenso wie die Parlamentsstellung. Die neue Verfassung der Russischen Föderation hat demgegenüber einen fast autokratischen Präsidenten; sie ist aber immerhin gesetzestechnisch etwas modemer und vermeidet mehr als die anderen Verfassungstexte einige formale Charakteristika sozialistischer Verfassungs- und Gesetzgebung.75 (Man darf sich fragen, ob diese Verbesserungen und Machumschichtungen Grund genug waren, unter dem etwas unüberlegten Beif all der westlichen Welt das Parlament zu stürmen - zumal man nun im Ergebnis der Wahlen ein ebenso radikales, aber direkt demokratisch legitimiertes Parlament hat.)

Ein weiteres Charakteristikum, das auf alle Verfassungen bzw. Verfassungsprojekte zutrifft, ist die Mühe, mit der man - wenn überhaupt - die unsichtbaren Schamierstellen westlicher Verfassungssysteme erkennt und regelt. So gehen etwa alle Verfassungen bzw. Verfassungsprojekte davon aus, daß ein Gesetzesvorbehalt als solcher eine ausreichende Grundrechtssicherung darstellt und daß es für alle Grundrechte mehr oder weniger die gleichen Schranken gibt: Zumindest in den Texten scheint das Wissen um die je spezielle Gefährdung von unterschiedlichen Grundrechten nicht durch. Auch der Gedanke, daß mit dem Gesetzesvorbehalt alleine und seiner wie auch immer spezifizierten Qualifizienung noch nicht viel erreicht ist, daß man vielmehr auch einen Maßstab für die Eingriffstiefe von

74 Art. 20 Abs. 2 VerfERF/OS; Art. 20 Abs. 2 nVerfRF, wobei sich der Staat nach der Version des Obersten Sowjets (und nach Art. 38 Satz 3 aVerfRF) wenigstens um die Abschaffung der Todesstrafe bemühte; Art. 8 VerfKas; Art. 16 VerfEst; Art. 21 Abs. 3 VerflEUkr; Art. 24 Abs. 2 LVerfLEWeißr; ohne Regelung nur Art. 24 VerfUs.

75 Etwa die Vermeidung langer Enumerativkataloge mit diese am Schluß ad absurdum führenden Generalkompetenzen oder die sparsame Verwendung des sinnlosen Zusatzes "im Rahmen ihrer Kompetenzen" für das Handeln von Staatsorganen, s. die Hinweise in Fn. 60. 
staatlichen Maßnahmen benötigt, hat sich bisher nur in der neuen Verfassung der Russischen Föderation bis hin in eine Formulienung durchgesetzt. 76

Weiter ist interessant, da $B$ der gesamte Bereich der rechtsstaatlichen Normenhierarchie und das Problem der Delegation von Rechtssetzungskompetenzen vom Parlament auf die Exekutive nirgendwo überzeugend geregelt ist. Das Verständnis des Gesetzesvorbehalts als Parlamentsvorbehalt im Bereich der Grundrechte verkennt schlicht, daß ein Parlament das alles regeln muß, überfordert ist, sowie auch die grundrechtliche Gefährlichkeit von exekutivischer Rechtsschöpfung: Dabei müßte gerade dies aus leidvoller Erfahrung den Verfassungsschöpfern im GUS-Land klargeworden sein.77 Da man die exekutivische Rechtsschöpfung offensichtlich nicht als rechtsstaatliches und grundrechtliches Problem zur Kenntnis nehmen will, überrascht es nicht, daß auch das Problem der Delegation von Rechtssetzungsbefugnissen des Parlaments auf die Exekutive nirgendwo geregelt ist: Dagegen findet sich in einigen Verfasssungstexten das Verbot einer Delegation präsidialer Dekretkompetenz auf andere Teile der Exekutive.78

In diesen Problembereich fallt auch die eigentlich interessante und auf den ersten Blick verständliche verbreitete Nutzung der franzßsischen Konzeption der "organischen Gesetze":79 Bei dieser Adaption entstand freilich kein organisches Gesetz im französischen Sinne. Im Regelfall handelt es sich vielmehr um mit qualifizierter Mehrheit zu verabschiedende Gesetze, die nicht Teil des Verfassungstextes sind; in etwas anderer Variation kennt die deutsche Rechtsgeschichte dies unter dem Begriff der "Verfassungsdurchbrechung"; der Grundgesetzgeber hat in Art 79 Abs. 1 GG mit dem Gebot der Textänderung solchen Verfassungsdurchbrechungen einen Riegel vorgeschoben. Bezüglich der Verwendung dieser Verfassungsgesetze in den Nachfolgestaaten der UdSSR erscheint eine gewisse Skepsis am Platze: Nachdem diese Rechtssysteme schon mit den einfachen Normenhierarchien nur sehr schwer klarkamen und klarkommen, wird eine weitere Stufe in dieser Normenhierarchie in Form des "Verfassungsgesetzes" die Dinge in Zukunft nicht vereinf achen.

76 Siehe Art. 55 Abs. 3 nVerfRF.

77 Siehe dazu Blankenagel, Rechtsstaat UdSSR, (Anm. 63), S. 21 ff.

78 Siehe z.B. Art. 79 Abs. 3 VerfKas: Delegationsverbot für den Präsidenten, soweit es um seine Machtbefugnisse als Staatsoberhaupt geht; Art. 93 Abs. 2 VerfUs enthält ein ähnliches Delegationsverbot; sehr global für den Präsidenten Art. 179 VerfEUkr, ebenso Art. 100 Abs. 2 VerfEWeißr.

79 Siehe Art. 108 nVerfRF, wobei der Präsident gegenüber Verfassungsgesetzen kein Vetorecht hat, Art. 108 Abs. 2 Satz 2; Beispiele siehe etwa in Ar. 114 Abs. 2, 118 Abs. 3; bei Art. 118 Abs. 3 der Regelung der Gerichtsbarkeit, sind im Hinblick auf die einf achen Regelungsvorbehalte des Art. 119 Abs. 2 sowie 120 Satz 1 ganz erhebliche Abgrenzungsprobleme zu erwarten. Siehe weiter Ar. 129 Abs. 3 sowie Art. 5 Übergangsbestimmungen VerfKas; Beispiele siehe in den Art. 4 Abs. 2, 62 Abs. 2, 69 Abs. 2, 74 Abs. 2; siehe weiter Art. 258 VerfEUkr, mit einer Aufzählung der Verfassungsgesetze in Art. 134 Abs. 2 VerfEUkr. 
Durfte bei Gesetzesvorbehalt und Delegation noch von nicht verstehender Blindheit ausgegangen werden, so ist in einem anderen Bereich schlicht vorsätzliches Augenschließen in Machtbereicherungsabsicht zu konstatieren: Alle Texte garantieren eine dürre Freiheit der Massenmedien. Bezüglich einer Entstaatlichung des staatlichen Rundfunks und Fernsehens herrscht verbissenes Schweigen; nicht einmal eine Trägervielfalt neben dem staatlichen Rundfunk und Fernsehen wird garantiert. Vor dem Hintergrund des seit Anfang 1993 in der Russischen Föderation ausgefochtenen Kampfes um das Femsehen sind diese Regelungen wohl eine vorläufige Absage an freie Funkmedien (bei den Zeitungen ist dies anders); der neueste Erlaß Jelzins bestätigt diese Einschätzung. 80

Neben diesen inhaltlichen Besonderheiten postsozialistischer Verfassungsgebung fallen noch zwei modale Charakteristika auf. Das eine könnte man als "planwirtschaftlichen approach" bezeichnen, mit anderen Worten als die Vermutung oder Hoffnung, daß die Wirklichkeit sich schon den normativen verfassungsrechtlichen Vorgaben, d.h. dem Plan, anpassen werde. Bezeichnend ist etwa, daB alle Verfassungsprojekte und Verfassungen sehr strenge Mehrheitsregeln haben. So ist der normale Abstimmungsmodus die Mitgliedermehrheit; der Rekord lag bei einer 3/4 Mehrheit der Mitglieder mit einem zusätzlichen sehr strengen Quorum.81 Historisch ist dies leicht erklärlich: Hohe Abstimmungsmehrheiten waren das wohlfeilste Gut im politischen System des Sozialismus. Niemand kommt für die Zukunft auf den Gedanken, daß auf diese Weise eine obstruktive Minderheit die Möglichkeit hat, über Nichtteilnahme an Abstimmungen das Zustandekommen gültiger Entscheidungen zu blockieren. In besonderem Maße gilt dies für Verfassungsänderungen: Uber anscheinend strenge Erfordemisse haben hier wohl die Verfassungsgeber sich selbst ein Denkmal mit langer Haltbarkeitsdauer setzen wollen, eine Don-Quichoterie angesichts der realen Tempi der Verfassungsänderung in diesen Ländem. Besonders schwierig, ja fast unmöglich ist im übrigen eine Verfassungsänderung nach der neuen Verfassung der Russi-

80 Ant. 29 Abs. 5 nVerfRF: Freiheit der Masseninformation / Zensurverbot; viel pluralismusfreundlicher war z.B. Art. 73 VerfERF/OS; die alte Verfassung RF enthielt keine Freiheit der Massenmedien. Siehe weiter Art. 33 Abs. 2 VerfWeißr, zu dem Kampf um die Massenmedien siehe die Gründung des föderalen Informationszentrums, VSNDVS RF 1992/52, Pos. 1647; zu den neuen, die Massenmedien direkt unter präsidiale Kontrolle nehmenden Ukaz siehe SZ v. 8/9.1.1994, S. 8. $\mathrm{Zu}$ dem Streit um die Massenmedien siehe weitere Nachweise bei: Blankenagel, Sichtbarer Dritter (Anmerkung 9), Fn. 12.

81 Art. 102 Abs. 3, 103 Abs.3, 105 Abs. 2, Abs. 4 nVerfRF: Mitgliedermehrheit als normaler Abstimmungsmodus; ähnlich Art. 87 Abs. 3 VerfERF/OS; Art. 83 VerfUs; Art. 85 VerfEWeißr, eine normale Abstimmungsmehrheit enthält dagegen Art. 73 VerfEst. - Noch strenger sind die Mehrheitserfordemisse bei den Verfassungsänderungen: siehe etwa Art. 129 VerfKas: $2 / 3$ bzw. 3/4, soweit es um die "Grundlagen der Verfassung" (das besondere Einleitungskapitel) geht; auch die neue Verfassung der Russischen Föderation errichtet hier in Ar. 135 ganz erhebliche Hindernisse: die Abschnitte 1, 2 u. 9 können nur durch eine verfassungsgebende Versammlung geändert werden und gelten damit als neue Verfasung; im übrigen gilt gem. Art. 137, 108 Abs. 2 das Erfordemis einer 3/4 Mehrheit im Rat der Föderation, einer 2/3 Mehrheit in der Duma. 
schen Föderation: Die extrem starke Position des Präsidenten in Verbindung mit den konkreten Personen, die in Zukunft für diese Präsidentschaft in Frage kommen, läßt hier noch viel Raum für Überraschungen.

Ein weiteres gemeinsames Merkmal aller Verfassungen bzw. Entwürfe ist ein sehr unbekümmerter Umgang mit der Gesetzessprache. So gibt es etwa ein ganzes Bündel von Begriffen, mit denen die rechtlichen Verpflichtungen und Bindungen des Staates gegenüber dem Bürger oder umgekehrt beschrieben werden. Das Recht ist im Angebot seiner Bauelemente hier, wie auch sonst, begrenzt: Primäre, sekundäre und außerrechtliche Leistungspflichten des Staates mit oder ohne korrespondierende subjektive Rechte des Bürgers: Der Reichtum der russischen Sprache überfährt hier die kargen Altemativen des Rechtssystems. Die gleiche wilde Sprache finden wir auch bei den unterschiedlichen Varianten von Mitwirkungs-, Zustimmungs-, Einspruchs- und Vetorechten im Zusammenwirken der Verfassungsorgane. Auch für diese wilde Sprache gibt es mehrere Erklärungen, die wohl kurnulativ heranzuziehen sind. Verfassungen wurden zum einen nie als geltendes Recht verstanden: Weshalb aber sollte man sich dann besonders bemühen - auch wenn man eigentlich weiß, daß dieser Zustand sich geändert hat (?) und man sich deswegen eigentlich mehr bemühen sollte. Darüber hinaus fallt es in der Regel den Autoren der Verfassungsentwürfe schwer, sich in die Situation eines Normanwenders hineinzuversetzen und die Norm auf Schwachstellen abzuklopfen: Die Autoren, zumeist Rechtswissenschaftler / Staatsrechtler, kennen aus ihrer beruflichen Praxis nicht die Situation, eine Norm, und sei es auch nur in der Lehre oder im Planspiel, anwenden zu müssen. Der dritte Grund schließlich ist ein kulturelles Spezifikum, das hier auch auf das Rechtssystem und die Formulierung der Verfassungen durchschlägt. Die sowjetische Gesellschaft lebte zu einem erheblichen Teil in Worten und Losungen; diese Losungen waren das Surrogat realer gesellschaftlicher Leistungen und Fortschritte. Das Wort wurde für die Tat genommen; die neue Tat, die Reform, der Umbruch war vor allem und zunächst immer auch das Wort, das zu ihrem Symbol wurde. Je größer und neuer die Worte, desto größer und neuer die Taten und gesellschaftlichen Reformen: Eine neue Verfassung ist also ohne neue, große Worte nicht denkbar und, problematischer noch, auch hier wird wahrscheinlich das neue Wort für die Tat der Schaffung eines Verfassungsstaates und einer mit einer Verfassung (und nicht gegen sie) lebenden Gesellschaft genommen.

\section{Ex constitutione lux}

Mit diesem problematischen Junktim von Wort und Tat stehen wir angesichts der neuen Verfassungen und Verfassungsprojekte auch unmittelbar vor der zentralen Frage: Dürfen wir nun von der Verfassungsgebung entscheidende Impulse für die Weiterentwicklung der von uns betrachteten Länder zu rechtsstaatlichen Demokratien erwarten, nach dem Motto, daß es egal ist, wo man in den Fluß springt, wenn man schwimmen lemen will. Die Ant- 
wort wäre, daß man in der Tat überall im Fluß - freilich besser oder schlechter - schwimmen lemen kann, wenn es denn wirklich darum geht, schwimmen zu lemen!

Es liegt auf der Hand, daß eine wirkliche gesellschaftliche Veränderung ohne eine grundlegende Reform des Wirtschaftssystems, ohne grundlegende Veränderungen in den Machtstrukturen des politischen Systems und ohne ebenso grundlegende Veränderungen in den Einstellungen der Gesellschaftsmitglieder nicht denkbar ist. In diesem Sinne ist Verfassungsgebung zunächst einmal ein bißchen Farbe an der Spitze des Eisbergs, die nichts oder wenig zu bewirken vermag und zu bewirken scheint. Dieser resignative Realismus hat vieles für sich; er verkennt, daB der Beginn der Reformen durch die Schaffung einer neuen Verfassung eventuell die konsensfähigste und kostengünstigste (und auch symbolstärkste) Alternative ist, die die neuen Staaten für den Reformprozeß haben. Die Wirtschaftsreformen, besonders die Privatisienung, sind sehr zeitaufwendig und kostenintensiv; sie sind es auch in dem Sinne, daß bei der Verteilung des Guts "Staatliche Wirtschaft" viele schwerwiegende materielle Interessen involviert sind und deren Träger entsprechend heftig und beharrlich versuchen werden, den Verteilungsproze $B$ zu ihren Gunsten zu beeinflußen. Entsprechend mühsam und langwierig wird der PrivatisierungsprozeB in Osteuropa sein; die bisherigen Erfahrungen bestätigen dies. Derngegenüber ist eine Verfassung kostengünstig und vermeintlich harmloser. Sie ist kostengtrnstig, weil es zunächst einmal "nur" darum geht, ein Gesetz zu machen; die Implementationskosten, etwa durch die Schaffung eines Verfassungsgerichts, folgen erst einige Zeit später. Sie ist vermeintlich harmloser als die Privatisierung, weil es anscheind nicht "wirklich" um reale Machtverteilung geht, da ja Verfassungen in der Erfahrung der von uns betrachteten Gesellschaften eigentlich immer nur auf dem Papier stehen. Insofern mag die Verfassungsgebung ein günstiger, leichter und unverdächtiger Einstieg in grundlegende gesellschaftliche Reformen sein.

Freilich muß der Einstieg oder der Wille, schwimmen zu lemen, emsthaft sein. Wird die Verfassung als Selbstverpflichtung nicht emst genommen oder nimmt die Gesellschaft die politischen Eliten nicht bei ihrem verfassungsrechtlichen Wort, so wird in kürzester Zeit die neue Verfassung zu einem Symbol unguter gesellschaftlicher Kontinuität, zu einem weiteren Beweis, daß wiederum nur ein Vorzeigepopanz, eine "pokazucha" geschaffen worden ist. Werden aber diese neuen Verfassungen emst genommen, so werden sie als sich selbst erfüllende Prophezeiung in ihren Konsequenzen auch sehr wirklich sein. Die neuen Verfassungen, so unser Resumé, könnten also ein kostengünstiger Einstieg in einen sehr langwierigen und schwierigen Prozeß sein, bei dem die dargestellten Gesellschaften und Staaten verfassungsrechtlich schwimmen lemen. Wenn es denn einer ausreichenden Menge von Akteuren darum geht: $\mathrm{DaB}$ gerade unüberlegtes Springen in einen Fluß für den Nichtschwimmer allemal eine gute Chance des Errinkens bereithält, bedarf keiner besonderen Klarstellung. 


\title{
ABSTRACTS
}

The Creation of Constitutions in the CIS-States - Awakening from Socialistic Sleep or Waking up of Socialistic Sleepers

\author{
By Alezander Blankenagel
}

The constitutional development in the CIS-States shows differing patterns, ranging from long lasting yet unsuccessfull attempts to have a constitution passed by the traditionallyoriented Supreme Soviets to the painful or painless passing of new constitutions. Most of the constitutions, with the notable exception of Estonia. follow basically the same pattern, showing a partial rejection and a partial acceptance of likewise socialist past and westem constitutional experience. The main differences can be found in the regulation of the division of powers between parliament and president. Not surprisingly all the constitutions show similar patterns of shortcomings which are partly due to an uncomplete understanding of the mechanics of constitutionalism; some of these shortcomings, though, can be attributed to a willful omission of very basic preconditions of the functioning of democracy in favor of a better grip of power for the leading elites. Having in mind that new constitutions are but one - and may be a not very important - element of the big societal reform, the success of all these constitutions will depend on the general approach of the elites and the population of the respective society: Only when taken seriously (and not considered to be some cheap symbol) they will have a chance to be a selffulfilling prophecy.

\section{The 1993 Vienna Declaration of Human Rights - New Perspectives for the Protection of Human Rights?}

By Philip Kunig and Robert Uerpmann

Although the protection of human rights has become a concem both of general and of regional intemational law, massive and continuous violations of human rights are still frequent. While political and economic developments raise new problems, the very foundations of international human rights law remain uncertain. 45 years after the adoption of the Universal Declaration of Human Rights, the 1993 World Conference on Human Rights was expected to assess present achievements and to draw perspectives for further improvements. However, serious political controversies occurred. The Conference's final document, the Vienna Declaration, does not present a coherent concept of human rights, of their foundations and universality. In the field of enforcement, the Declaration lays out a complex protection system based on the cooperation of various institutions. It relies on the 